\title{
Abnormal Lysosomal Positioning and Small Extracellular Vesicle Secretion in Arterial Stiffening and Calcification of Mice Lacking Mucolipin 1 Gene
}

\author{
Owais M. Bhat ${ }^{1}$, Xinxu Yuan ${ }^{1}$, Sarah Camus ${ }^{1}$, Fadi N. Salloum ${ }^{2}$ and Pin-Lan Li ${ }^{1, *}$ \\ 1 Department of Pharmacology and Toxicology, School of Medicine, Virginia Commonwealth University, \\ Richmond, VA 23298, USA; Owais.Bhat@vcuhealth.org (O.M.B.); xinxu.yuan@vcuhealth.org (X.Y.); \\ Sarah.Camus@vcuhealth.org (S.C.) \\ 2 VCU Pauley Heart Center, Division of Cardiology, Department of Internal Medicine, \\ Virginia Commonwealth University, Richmond, VA 23298-0204, USA; fadi.salloum@vcuhealth.org \\ * Correspondence: pin-lan.li@vcuhealth.org; Tel.: +1-804-828-4793; Fax: +1-804-828-4794
}

Received: 31 December 2019; Accepted: 28 February 2020; Published: 3 March 2020

\begin{abstract}
Recent studies have shown that arterial medial calcification is mediated by abnormal release of exosomes/small extracellular vesicles from vascular smooth muscle cells (VSMCs) and that small extracellular vesicle (sEV) secretion from cells is associated with lysosome activity. The present study was designed to investigate whether lysosomal expression of mucolipin-1, a product of the mouse Mcoln 1 gene, contributes to lysosomal positioning and sEV secretion, thereby leading to arterial medial calcification (AMC) and stiffening. In $M c o l n 1^{-1-}$ mice, we found that a high dose of vitamin D (Vit D; 500,000 IU/kg/day) resulted in increased AMC compared to their wild-type littermates, which was accompanied by significant downregulation of SM22- $\alpha$ and upregulation of RUNX2 and osteopontin in the arterial media, indicating a phenotypic switch to osteogenic. It was also shown that significantly decreased co-localization of lysosome marker (Lamp-1) with lysosome coupling marker (Rab 7 and ALG-2) in the aortic wall of $M c o l n 1^{-/-}$mice as compared to their wild-type littermates. Besides, Mcoln $1^{-1-}$ mice showed significant increase in the expression of exosome/ sEV markers, CD63, and annexin-II (AnX2) in the arterial medial wall, accompanied by significantly reduced co-localization of lysosome marker (Lamp-1) with multivesicular body (MVB) marker (VPS16), suggesting a reduction of the lysosome-MVB interactions. In the plasma of $M c o l n 1^{-1-}$ mice, the number of sEVs significantly increased as compared to the wild-type littermates. Functionally, pulse wave velocity (PWV), an arterial stiffening indicator, was found significantly increased in Mcoln $1^{-/-}$mice, and Vit D treatment further enhanced such stiffening. All these data indicate that the Mcoln1 gene deletion in mice leads to abnormal lysosome positioning and increased sEV secretion, which may contribute to the arterial stiffness during the development of AMC.
\end{abstract}

Keywords: arterial medial calcification; $M c o l n 1^{-/-}$, multivesicular body; smooth muscle cells; small extracellular vesicle

\section{Introduction}

Vascular calcification (VC) was considered to be a passive and degenerative process, whereas now it is considered to be an actively regulated process associated with the pathological deposition of calcium minerals in the vascular system [1,2]. The intimal calcification is associated with an increased deposit of lipids, and inflammatory cell infiltrate, while medial calcification involves a phenotypic transformation of vascular smooth muscle cells (VSMCs) in the arterial media [3,4]. Medial calcification is commonly found in patients with advanced age, diabetes, calcific aortic valve disease, chronic kidney disease (CKD), and chronic inflammatory diseases [5]. One of the possible mechanisms of VC is phenotypic 
change of VSMCs from a contractile to a synthetic and osteochondrogenic phenotype followed by augmented expression of osteochondrogenic markers, for example, osteocalcin, osteopontin, RUNX2, and alkaline phosphatase and reduced expression of VSMC contractile markers such as smooth muscle $\alpha$-actin and smooth muscle $22 \alpha$ (SM22- $\alpha$ ). Another proposed mechanism is the release of matrix vesicles from viable VSMCs to initiate the VC [6].

Exosomes (40-140 nm in size) are a group of extracellular vesicles (EVs) with intracellular contents, such as proteins and RNAs [7] that are associated with VC [8,9]. Exosomes are secreted by VSMCs in vivo after pro-calcifying stimulation and become calcified to initiate VC [8]. Recent investigations revealed that an increase in exosomes secreted by VSMCs promotes VC via mineral deposition. Additionally, exosomes derived from calcified VSMCs were demonstrated to enhance calcification in the recipient VSMCs through initiating mitogen-activated protein kinase [10]. It is reported that the exosome secretion pathway is activated during $\mathrm{VC}$, and modulations on such a pathway may be applied as novel approaches for VC prevention [8]. During VC progression, the phenotypic transition process involves cytoskeleton remodeling, such as intracellular modifications, which enhanced exosome secretion [11,12].

Lysosome coupling or trafficking is a crucial process to perform the lysosome fusion and fission events $[13,14]$. Various proteins are involved in lysosome coupling such as dynein-dynactin, Rab proteins, ALG-2, Arl8, etc., which in association with SNARE proteins are essential for the fusion/fission process. Rab7, a small GTPase, and lysosomal $\mathrm{Ca}^{2+}$-sensor ALG-2 (also known as PDCD6) are known to play essential roles in the lysosome coupling process [15]. Belonging to the large family of transient receptor potential ion channels, lysosome Transient receptor potential mucolipin 1 (encoded by the Mcoln1 gene, also known as TRPML1) [16,17] permeates $\mathrm{Ca}^{2+}, \mathrm{Na}^{+}$, and other cations [18-20]. Mutations in the TRPML1 gene lead to mucolipidosis type IV (ML4) disease in humans, which is characterized by defects in membrane trafficking in the late endocytic pathway due to enlarged lysosomes [16,21] and impaired lysosome biogenesis [22,23]. In TRPML1 ${ }^{-/-}$osteoclasts, markedly enlarged lysosomes were observed as occurring in lysosomal storage diseases [24], which lead to defective lysosomal trafficking.

It has been reported that TRPML1 plays a critical role in lysosomal trafficking and various other lysosomal functions [25,26]. Mucolipin 1, also known as TRPML1, in association with Rab7, ALG-2, and other proteins, regulates centripetal transport of lysosomes crucial for lysosome exocytosis in response to changes in $\mathrm{Ca}^{2+}$ levels [15]. TRPML1 induces perinuclear redistribution of lysosomes, then links with ALG-2, which consequently causes TRPML1-dependent retrograde transport of lysosomes [27]. Based on earlier studies, multivesicular bodies (MVBs) and lysosome fusion are critical for the lysosomal degradation of MVBs [28,29]. Recently, a study and ours, also demonstrated that TRPML1 regulates lysosome fusion with MVBs and the plasma membrane which contributes to exosome release [30]. Our findings in podocytes demonstrated that inhibition or activation of the TRPML1 channel modulates exosome release from these cells [31]. Recently, we observed that inhibition of the TRPML1 channel by increased production of lysosomal sphingolipids due to acid ceramidase deficiency in arterial smooth muscle cells (SMCs) prevented lysosome-MVB interactions which resulted in enhanced small extracellular vesicle (sEV) secretion triggering arterial medial calcification (AMC) (unpublished data). Although, there is no direct evidence about whether Mcoln1 is associated with arterial stiffness in vivo. Several studies revealed that transient receptor potential (TRP) channels contribute to the VSMC phenotypic transition and vasoconstriction, also release of calcified vesicles from these VSMCs [8,32], which are features that are involved in arterial stiffness during the progression of AMC [33].

Based on this knowledge, the present study investigated whether Mcoln1 gene deletion enhances phenotypic switch in arterial medial SMCs in a mouse model of AMC. We further explored whether Mcoln 1 gene deletion contributes to impaired lysosome positioning-induced sEV secretion in arterial medial SMCs, which may implicate a mechanistic pathway leading to AMC and arterial stiffening. Our data suggest that Mcoln1 deficiency impairs lysosomal positioning, which may prevent 
lysosome-MVB interaction and increase sEV secretion. Besides, we also determined that the Mcoln 1 gene contributes to phenotype change and arterial stiffness in vivo.

\section{Results}

\subsection{Characterization of Mucolipin 1 (Mcoln1) Gene KO Mice}

To characterize the mice, polymerase chain reaction (PCR) was used to verify the mice homozygous for the Mcoln 1 gene deletion. As shown in Figure 1A, Mcoln $1^{-/-}$has only mutant $750 \mathrm{bp}$ PCR product while the heterozygous has both the mutant $750 \mathrm{bp}$ and WT $453 \mathrm{bp}$ PCR products. Wild-type mice had only the $453 \mathrm{bp}$ PCR product. RNA was isolated from aortic tissues to analyze the mRNA expression of TRPML1, also known as Mcoln1 gene, in both wild-type and Mcoln1 $1^{-/}$mice. RT-PCR shows Mcoln1 gene deletion in $\mathrm{Mcoln}^{-/-}$mice as compared to wild-type mice, as shown in Figure 1B. Immunostaining was used to confirm the expression of mucolipin 1 in aortic sections to validate Mcoln 1 gene deletion; we found positive immunostaining of mucolipin 1 in WT/WT mice, which was absent in Mcoln $1^{-/-}$ mice as shown in Figure 1C.

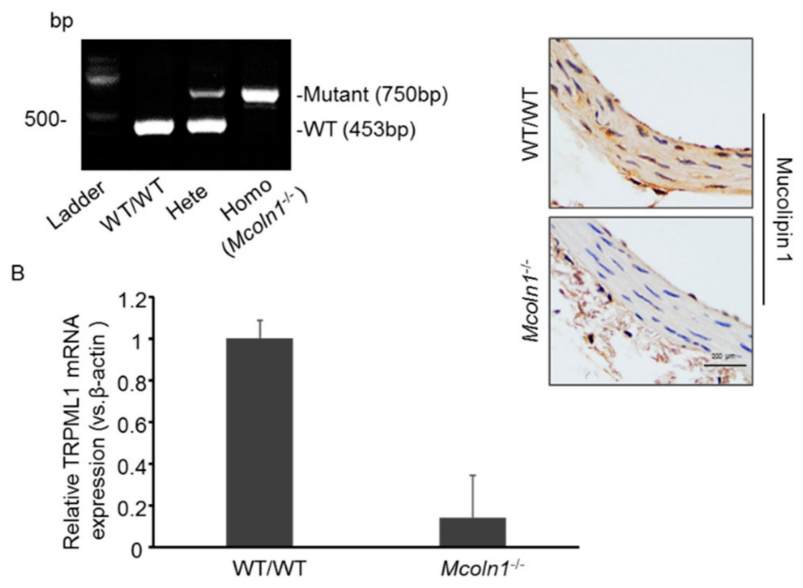

Figure 1. Characterization of Mcoln $1^{-/-}$mice. (A) Mucolipin $1 \mathrm{KO}$ (Homozygous, Mcoln $1^{-/-}$) had one positive PCR product for mutant $750 \mathrm{bp}$. Heterozygous mice had two positive PCR products including $750 \mathrm{bp}$ for mutant and $453 \mathrm{bp}$ for WT/WT. WT/WT mice only had wild-type Mcoln1 gene (453 bp). (B) Mcoln1 gene deletion was validated by RT-PCR as depicted by mRNA expression of TRPML1 gene, also known as Mcoln1. Data are shown as mean \pm SEM of values. $n=3$. (C) Representative immunohistochemical images of aortic sections reveal abolished immunostaining of mucolipin 1 (brown stain) in $M c o l n 1^{-/-}$mice as compared to wild-type. $n=3$. bp: Base Pair; Homo: homozygous; Hete: heterozygous.

\subsection{Mcoln1 Gene Deletion Enhanced Arterial Medial Calcification in Vit D-Treated Mice}

To investigate the role of $M c o l n 1$ gene in AMC in vivo, subcutaneous (s.c.) injections of vitamin $\mathrm{D}$ (Vit D) were given to mice for four consecutive days. Calcium deposition was visualized in aortic cross sections by Alizarin Red staining. As shown in Figure 2A, representative images show markedly increased calcium deposition as reddish areas in the aortic media wall of $M c o l n 1^{-/}$mice as compared to their wild-type littermates. Moreover, we found significant changes in plasma calcium levels among treatments (WT and $M c o l n 1^{-/-}$vehicle $\sim 2.9 \mathrm{Mm}$, and WT and $M c o l n 1^{-/-}$Vit D 3.69 mM) but there was a non-significant increase among genotypes (WT and $M c o l n 1^{-/-}$mice). Furthermore, in coronary arteries, the photomicrographs show prominently increased calcium deposition due to Mcoln 1 gene deletion in $M c o l n 1^{-/-}$mice as compared to wild-type mice, as shown in Figure 2B. The summarized data in bar graph form are shown in Figure 2C,D. These results indicated that Mcoln 1 gene contributes to the AMC. 
A
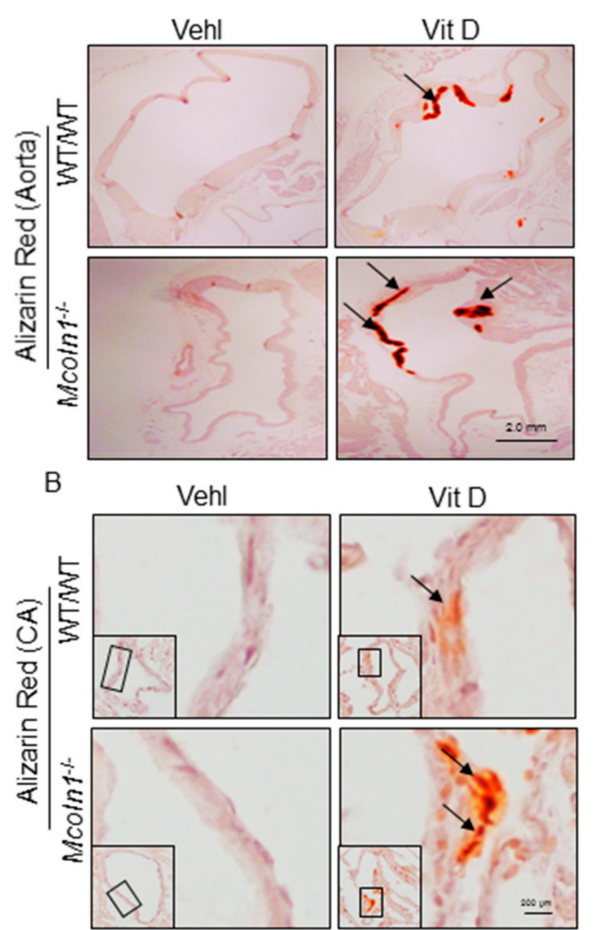

C

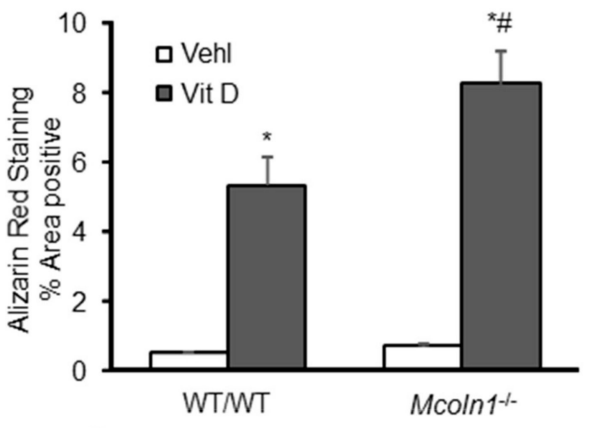

D

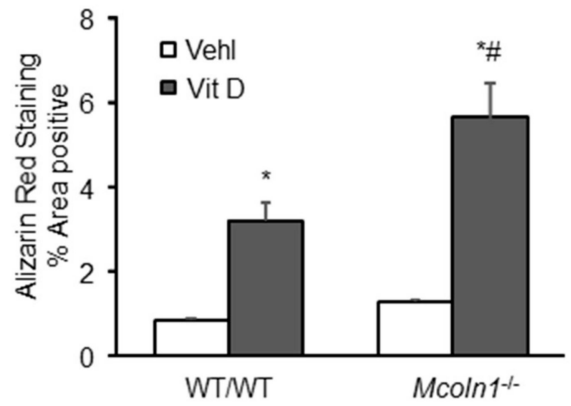

Figure 2. Arterial calcification in Vit D-treated $M \operatorname{coln} 1^{-/}$mice. Representative images of (A) Aortic and (B) Coronary artery sections stained by Alizarin Red S (red color) staining show significantly increased calcification in the aortic $(n=5-6)$ and coronary arterial $(n=5)$ medial wall of Vit D-treated Mcoln $1^{-1-}$ mice as compared to their littermates. (C,D) Summarized data showing calcification in the aortic and coronary arterial media. Data are shown as mean \pm SEM of values. Vehl: Vehicle; Vit D: vitamin D; CA: coronary artery. ${ }^{*} p<0.05$ vs. WT/WT Vehl; $\# p<0.05$ vs. WT/WT Vit D group.

\subsection{Arterial Medial SMC Phenotypic Transition in Vit D-Treated Mcoln $1^{-/}$Mice}

Smooth muscle cells are involved in vascular calcification and their phenotypic change from contractile to osteogenic is depicted by the development of mineralization, calcification prone matrix downregulation of SMC markers (SM22 $\alpha$ and SM $\alpha$-actin), and upregulation of osteochondrogenic markers such as RUNX2, osteopontin (OSP), osteocalcin, alkaline phosphatase (ALP) [34], etc. To determine whether Mcoln1 gene deletion is associated with phenotype change of SMC to osteogenic in AMC, immunostaining for SM22 $\alpha$, OSP, and RUNX2 was performed in the aortic sections. Under vehicle conditions, we observed a non-significant decrease in SMC contractile marker SM22- $\alpha$ in the aortic medial wall of Mcoln $1^{-/-}$mice as compared to their wild-type littermates, which was significantly decreased within the calcified areas after receiving Vit D treatment (Figure 3A,D). Moreover, the osteogenic markers such as OSP (Figure 3B) and RUNX2 (Figure 3C) were markedly increased in the arterial medial wall of Vit D-treated $M c o l n 1^{-1-}$ mice compared with their wild-type littermates. The summarized data are shown as bar diagrams (Figure 3E,F). 

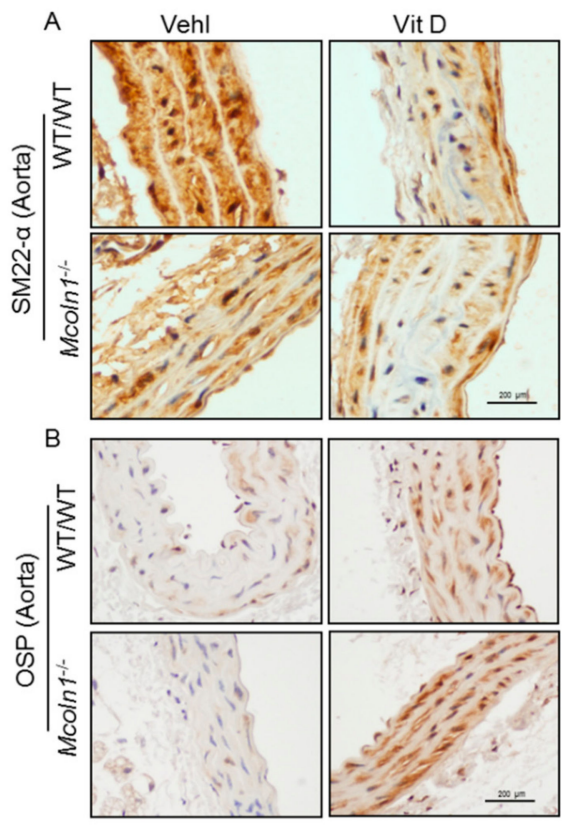

D
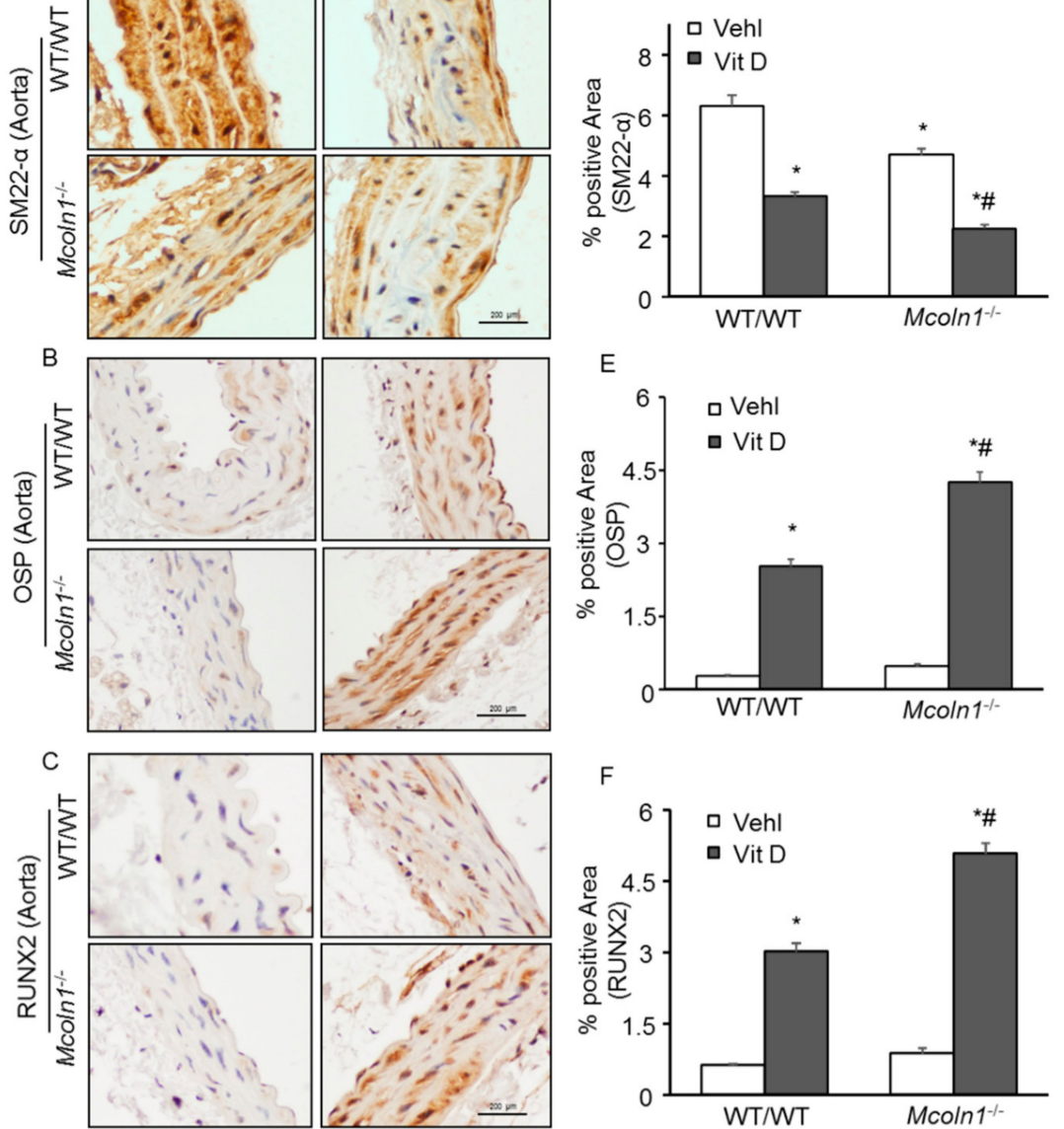

Figure 3. Phenotypic switch of smooth muscle cells (SMCs) in the aortic media in Vit D-treated Mcoln1 $1^{-/-}$ mice. Representative aortic photomicrographs depict arterial medial calcification (AMC) associated with (A,D) decreased expression of SM22- $\alpha$ (brown stain) $(n=5-6)$; (B,E) increased expression of OSP $(n=6)$; and $(\mathbf{C}, \mathbf{F})$ RUNX2 $(n=6)$ (brown stain) in the aortic media of Mcoln1-l- mice receiving Vit $\mathrm{D}$ injections as compared to wild-type littermates. Data are shown as mean \pm SEM of values. Vehl: Vehicle; Vit D: vitamin D; OSP: Osteopontin; RUNX2: Runt-related transcription factor $2 .{ }^{*} p<0.05$ vs. WT/WT Vehl; \# $p<0.05$ vs. WT/WT Vit D group.

In addition, RNA was isolated from aortic tissues and, using RT-PCR, we demonstrated that Mcoln1 gene deletion induced augmented mRNA expression of OSP and RUNX2 in Vit D-treated mice, as shown in Figure 4A,B. Also, Western blot analysis (Figure 4C) from aortic tissue protein lysates showed significant decrease in protein expression of SM22- $\alpha$, while an increased expression of RUNX2 was observed as shown in the bar graph (Figure 4D,E). The changes were more prominent with Mcoln 1 gene deletion in Vit D-treated Mcoln1 $1^{-/}$mice as compared to WT/WT mice. These results revealed that Mcoln 1 gene might be associated with phenotype change of arterial medial SMCs during the development and progression of AMC. Moreover, we observed decreased immunostaining of SM22 $\alpha$ (Figure 5A) in the coronary arterial wall associated with increased expression of OSP (Figure 5B) and RUNX2 (Figure 5C) in Vit D treated Mcoln1 ${ }^{-/-}$mice as compared to their wild-type littermates, as shown in Figure 5D-F. 
A

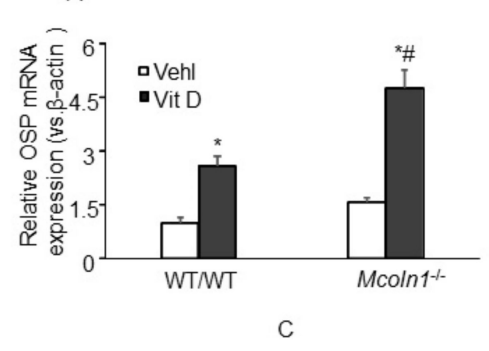

B

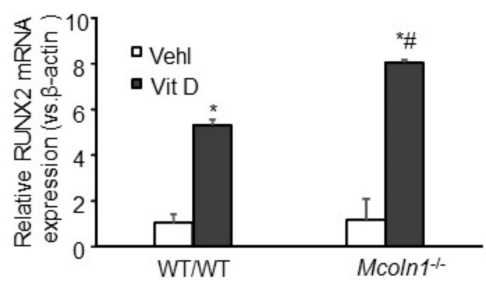

SM22- $\alpha$

RUNX2

$\beta$-actin

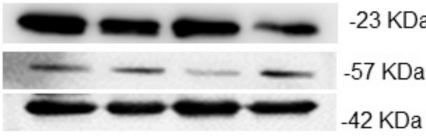

$\frac{\text { Vehl Vit D }}{\text { WTMT }} \quad \frac{\text { Vehl Vit D }}{M \operatorname{coln} 1^{1 /}}$
KDa
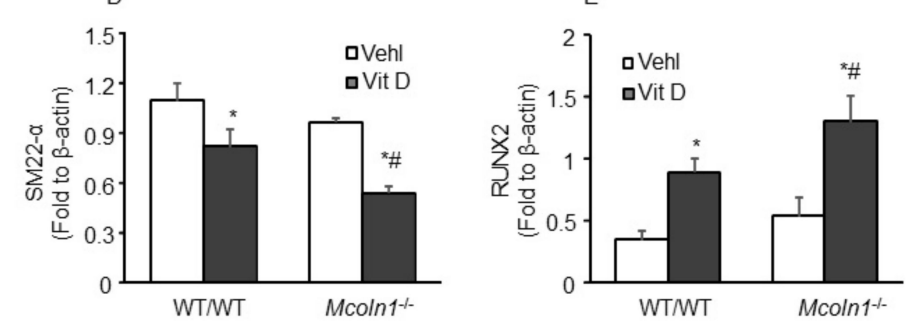

Figure 4. mRNA and protein expression of phenotypic switch markers in Vit D-treated Mcoln1 ${ }^{-/-}$mice. The bar graph shows significantly increased aortic mRNA expression of osteogenic markers (A) OSP and (B) RUNX2 in Mcoln1 $1^{-/}$mice receiving Vit D injections as compared to wild-type littermates. $n=3-4$. (C) Representative Western blot analysis showing the effects of Mcoln1 gene deletion on SM22- $\alpha$ and RUNX2 protein expression. Summarized data show the changes in the protein expression of (D) SM22- $\alpha$ and (E) RUNX2. $(n=3-4)$. Data are shown as mean \pm SEM of values. Vehl: Vehicle; Vit D: vitamin D; OSP: Osteopontin; RUNX2: Runt-related transcription factor $2 .{ }^{*} p<0.05$ vs. WT/WT Vehl; \# $p<0.05$ vs. WT/WT Vit D group. 

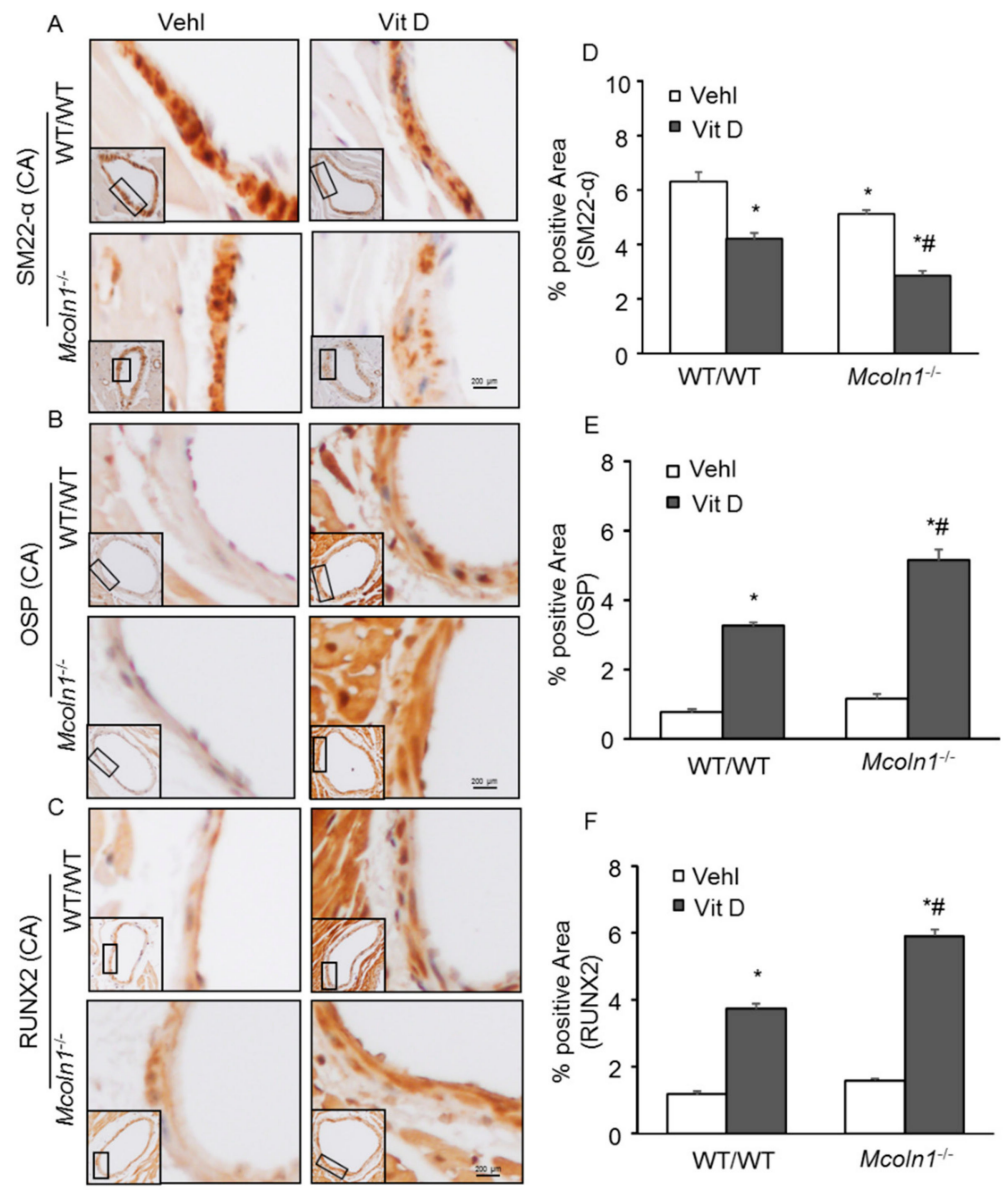

Figure 5. Phenotypic switch of SMCs in the coronary arterial wall in Vit D-treated $M c o l n 1^{-/-}$mice. Representative immunohistochemical images of coronary artery sections show decreased expression of (A) SM22- $\alpha$ (brown stain) $(n=5)$ and increased expression of (B) OSP $(n=5)$; and (C) RUNX2 $(n=5)$ (brown stain) in the coronary arterial wall of Vit D-treated Mcoln $1^{-/}$mice as compared to their littermates (D-F). The data of SMC phenotypic switch are summarized in the bar graph. Data are shown as mean \pm SEM of values. Vehl: Vehicle; Vit D: vitamin D; SM22- $\alpha$ : smooth muscle cell marker; OSP: Osteopontin; RUNX2: Runt-related transcription factor 2. ${ }^{*} p<0.05$ vs. WT/WT Vehl; \# $p<0.05$ vs. WT/WT Vit D group.

2.4. ALG-2 and Rab7 Interact with the Lysosome to Mediate Mcoln1-Dependent Lysosome Coupling in Aortic Medial SMCs in Vit D-Treated Mcoln1 ${ }^{-1-}$ Mice

Given the roles of lysosomal TRPML1 and the $\mathrm{Ca}^{2+}$ sensor ALG-2 in the process of lysosome coupling to dynein [15], and Rab7-dependent dynein-dynactin recruitment to late endosomes and lysosomes [27], we determined the role of ALG-2 and Rab7 on lysosomal positioning in AMC. We performed confocal microscopy of both ALG-2 and Rab7 and analyzed their co-localization with lysosome marker (Lamp-1). Due to Mcoln1 gene deletion, decreased co-localization of lysosome marker Lamp-1 (red) with lysosome coupling marker Rab 7 (green) (Figure 6A) and ALG-2 (green) (Figure 6B) was observed in the aortic medial wall of $M c o l n 1^{-/-}$mice as compared to wild-type mice, suggesting anterograde movement of lysosomes because of Mcoln 1 gene deficiency. The summarized data are shown as the Pearson correlation coefficient in Figure 6C,D. Hence, these results conclude that ALG-2 and Rab7 in association with TRPML1 promote perinuclear distribution of lysosomes required for the normal degradation process of lysosomes and other cellular processes. 

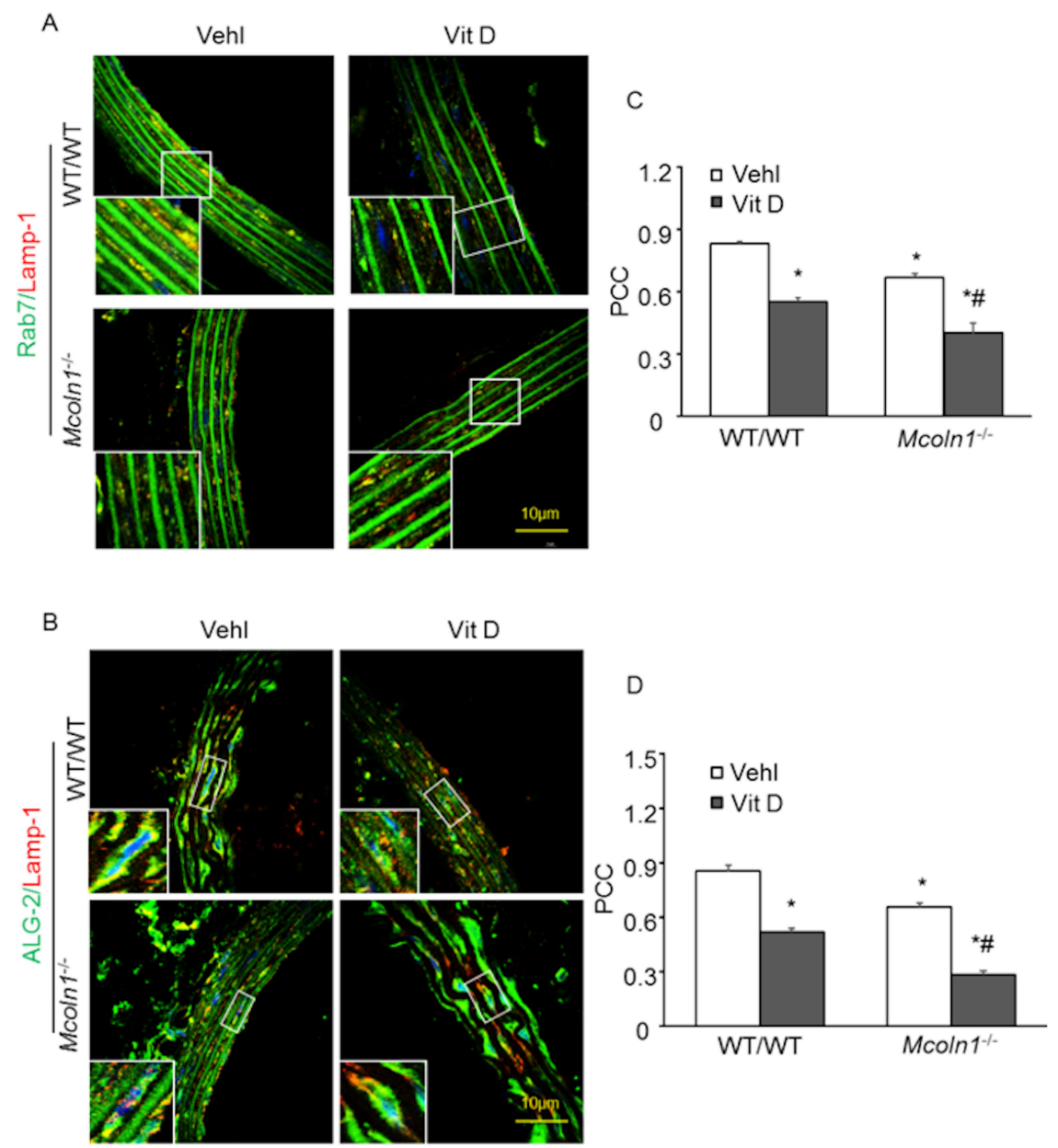

Figure 6. ALG-2 and Rab7 in association with lysosomes promote lysosome coupling in Vit D-treated Mcoln $1^{-/-}$mice. Representative confocal microscopic images depict co-localization of lysosome coupling markers (A) Rab7 (green), (B) ALG-2 (green), and lysosomes (lysosome marker Lamp-1; red) in aortic medial SMCs was much lower in Mcoln1 $1^{-1-}$ mice than their wild-type littermates receiving Vit D injection (C,D). The co-localization coefficient (PCC) as shown in the bar graph. Data are shown as mean \pm SEM of values. $n=5$. Vehl: Vehicle; Vit D: vitamin D; PCC: Pearson correlation coefficient. * $p<0.05$ vs. WT/WT Vehl; \# $p<0.05$ vs. WT/WT Vit D group.

\subsection{Mcoln1 Gene Deletion Elevated sEV Secretion by Inhibiting Lysosomes and MVB Interactions in the Arterial Medial Calcification}

Fusion of late endosomes with lysosomes leads to degradation of the endosomal contents. Moreover, fusion of late endosomes with the plasma membrane releases $40-140 \mathrm{~nm}$ vesicles known as exosomes in the outer space of the cell [35]. New molecular mechanisms provided evidence suggesting that SMC-derived extracellular vesicles (EVs), or exosomes, played a crucial role in arterial medial calcification [8]. Under mineral imbalance, VSMCs released calcified EVs that form microcalcification [32]. Therefore, using confocal microscopy we also observed the interaction of lysosomes and MVBs in aortic medial SMCs, which confirms lysosome trafficking and fusion to MVBs. Mice were treated with a high dose of Vit $\mathrm{D}$ to cause mineral imbalance. As shown in Figure 7A, co-localization of lysosome marker (Lamp-1; Alex 555, red fluorescence) and MVB marker (VPS16; Alex 488, green fluorescence) produced yellow puncta, indicating the lysosome-MVB interaction. Compared with the control wild-type littermates, Vit D treatment markedly decreased co-localization of Lamp-1 and VPS16 in arterial medial SMCs. Mcoln 1 gene deletion in the mice receiving Vit D injection caused a further decrease in the co-localization of Lamp-1 and VPS16 in aortic medial SMCs, as shown in the bar diagram (Figure 7B). 

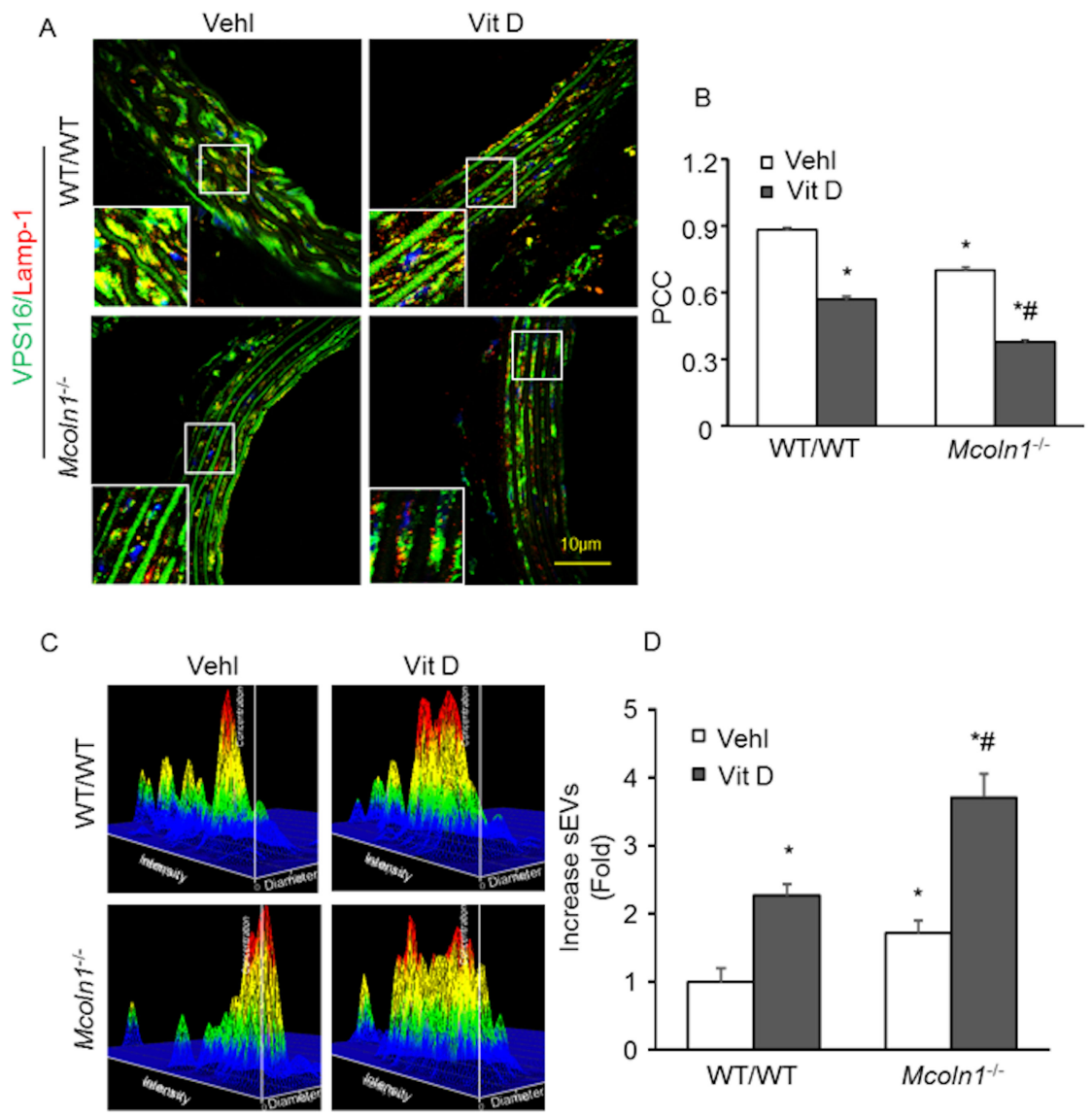

Figure 7. Lysosome-multivesicular body (MVB) interaction and small extracellular vesicle (sEV) release in Vit D-treated Mcoln1 $1^{-/}$mice. (A) Representative confocal pictures reveal co-localization of MVB (VPS16; green) and lysosome (Lamp-1; red) in aortic medial SMCs (B) Bar graph shows significant decrease in co-localization of VPS16/Lamp-1 in Vit D-treated Mcoln1-/- mice as compared to wild-type littermates. $n=5-6$. (C) Representative 3D graphs depicting number of sEVs isolated from plasma from wild-type and Mcoln1 $1^{-/-}$mice with vehicle and Vit D treatment. (D) Summarized bar graph shows changes in sEV number $(50-150 \mathrm{~nm})$ under vehicle and Vit D treatment. $n=5-7$. Data are shown as mean \pm SEM of values. Vehl: Vehicle; Vit D: vitamin D; PCC: Pearson correlation coefficient. * $p<0.05$ vs. WT/WT Vehl; \# $p<0.05$ vs. WT/WT Vit D group.

Furthermore, by immunohistochemistry, we found significantly increased levels of CD63 (Figure 8A) and annexin-II (AnX2) (Figure 8B) as exosome/sEV markers in the coronary arterial wall of Vit D-treated $M c o l n 1^{-/}$mice compared to the levels of their wild-type littermates. The summarized data are shown as bar diagrams in Figure 8C,D. These results indicate that Mcoln1 plays an important role in the fusion of lysosomes and MVBs, and Mcoln1 gene deletion impairs this fusion process and increases sEV release from arterial medial SMCs during AMC. 

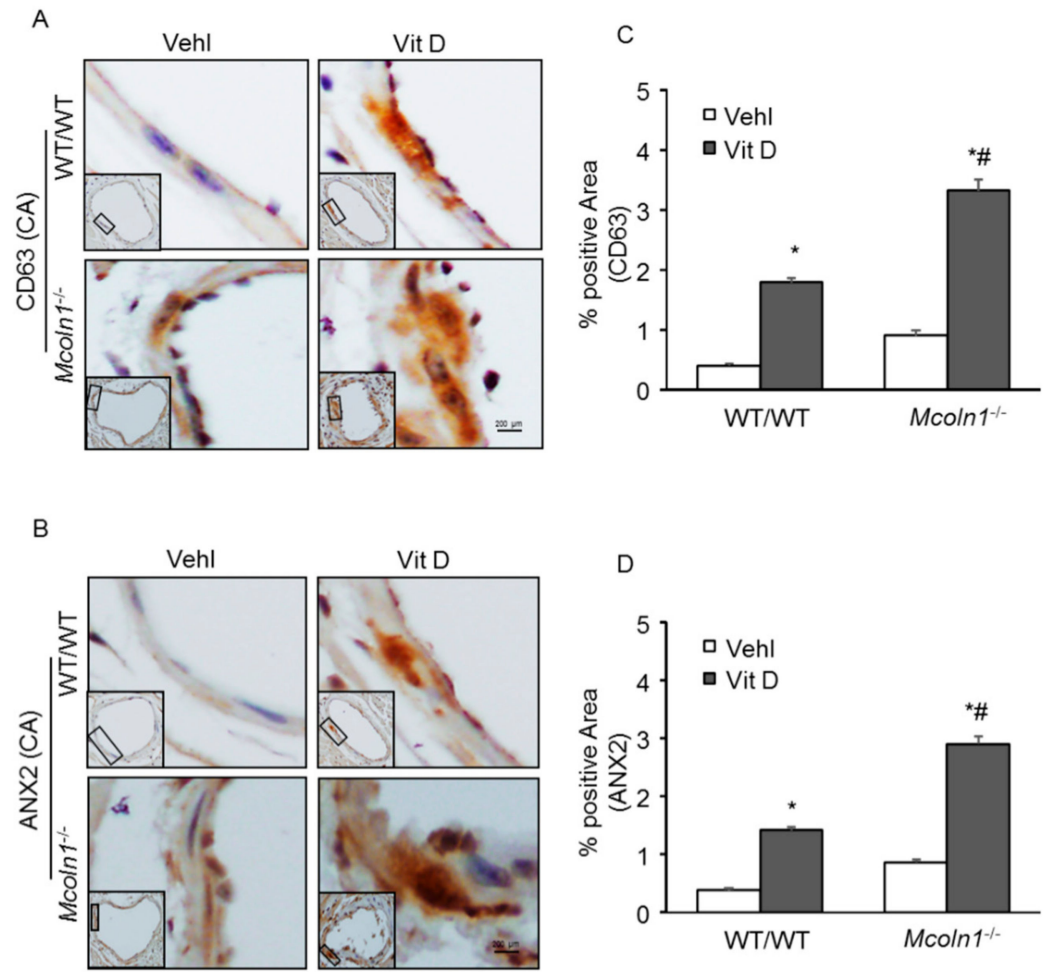

Figure 8. Localization of sEV markers in coronary arterial wall in Vit D-treated Mcoln $1^{-/-}$mice. Representative photomicrographs show immunostaining of sEV makers in coronary arterial wall. (A) CD63 and (B) AnX2 staining are observed as brown signal in the coronary arterial wall. The summarized bar graph shows \% positive area of (C) CD63 and (D) AnX2 positive staining in coronary arterial wall. Data are shown as mean \pm SEM of values. $n=5-6$. Vehl: Vehicle; Vit D: vitamin D; AnX2: Annexin-II; PCC: Pearson correlation coefficient. ${ }^{*} p<0.05$ vs. WT/WT Vehl; $\# p<0.05$ vs. WT/WT Vit D group.

\subsection{Role of Mcoln1 in sEV Release in the Arterial Medial Calcification}

To test whether Mcoln 1 controls MVB fate and sEV release in the AMC, we performed nanoparticle tracking analysis (NTA) to measure the number of small extracellular vesicles (sEVs) released in the plasma of the mice treated with a high dose of Vit D. Both wild-type and Mcoln $1^{-/-}$mice received Vit $\mathrm{D}$ injections to develop AMC. The representative 3-D histograms of vesicles showed the increased release of sEVs (50-150 $\mathrm{nm}$ in size) due to Mcoln 1 gene deletion in the plasma of Mcoln $1^{-/-}$mice even without any treatment, as shown in Figure 7C. Additionally, mineral imbalance due to high doses of Vit D-enhanced sEV release in the plasma of $\mathrm{Mcoln}^{-/-}$mice as compared to their wild-type littermates. The bar graph represents the vesicle counts (50-150 nm in size), as shown in Figure 7D. These results suggest that Mcoln1 is associated with the mechanistic pathway that mediates lysosome positioning and fusion to MVBs and controls MVB fate and sEV release.

\subsection{Lysosome-MVB Interactions and sEV Release Controlled by TRPML1 Signaling Pathway in Coronary Artery Smooth Muscle Cells (CASMCs)}

Further using wild-type CASMCs, we tested whether TRPML1 activation or inhibition affects lysosome-MVB interaction and sEV release. Prior treatment with TRPML1 agonist ML-SA1 prominently increased co-localization of VPS16 (MVB marker; green) and Lamp-1 (lysosome marker; red), whereas blockade of TRPML1 by verapamil (Vera) markedly decreased co-localization of VPS16 with Lamp-1 in these cells treated with or without Pi (Figure 9A). Quantitative analysis showed that the PCC of VPS16 with Lamp-1 increased in CASMCs treated with ML-SA1 and decreased by Vera (Figure 9B). Further, we observed that secretion of sEVs $(<200 \mathrm{~nm})$ were significantly decreased by ML-SA1 in Pi-treated 
CASMCs, as shown in Figure 9C,D. However, Vera significantly increased sEV secretion even under vehicle conditions, and caused further increase in Pi-treated CASMCs. These results suggest that Mcoln1 is important in the regulation of lysosome-MVB interactions and SEV secretion.

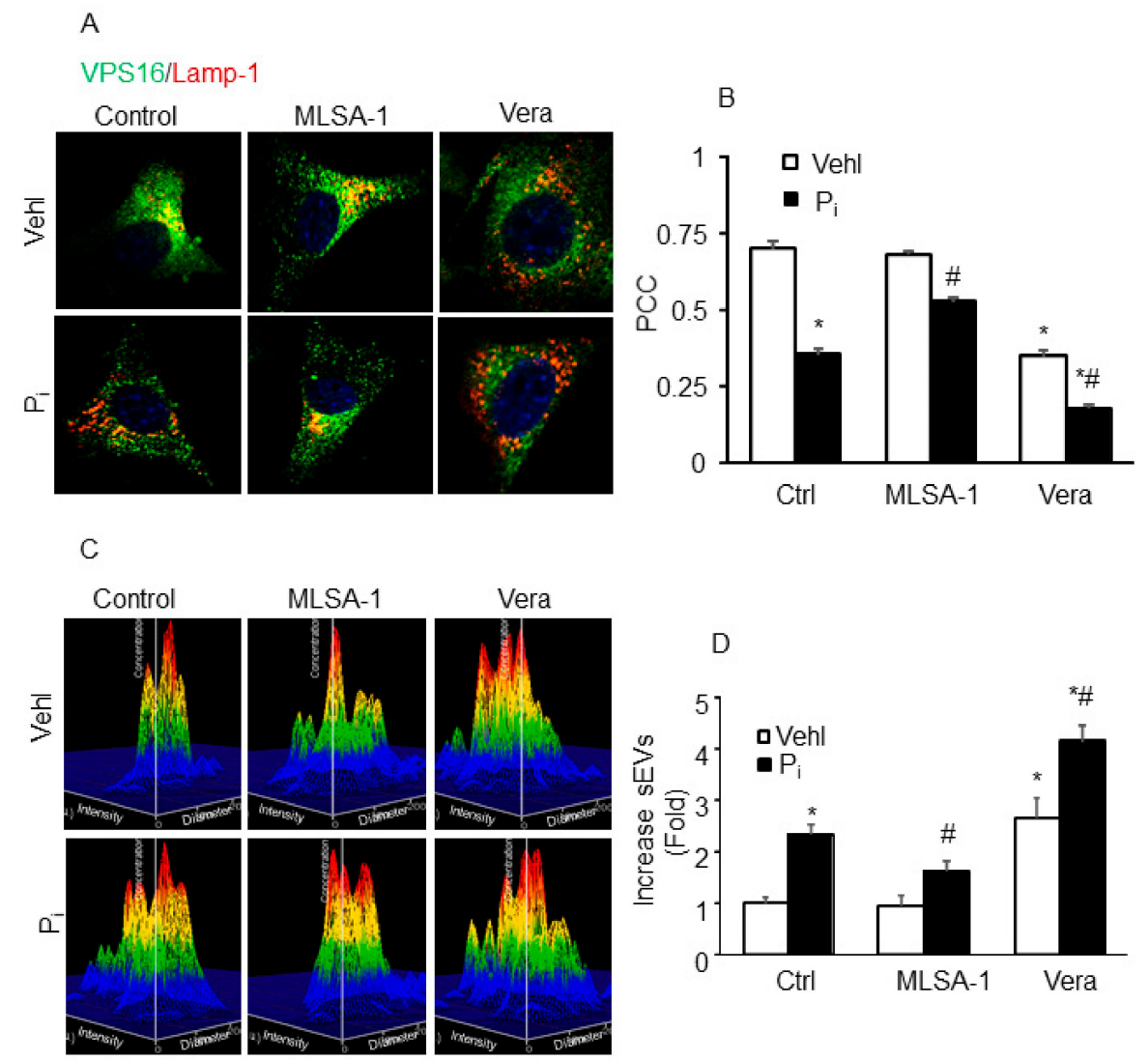

Figure 9. Effects of MLSA-1 and verapamil on lysosome-MVB interactions and SEV release in the wild-type CASMCs in vitro. (A) Representative confocal images show co-localization of VPS16 (green) and Lamp-1(red) in CASMCs. (B) Bar graph shows ML-SA1 $(10 \mu \mathrm{m})$ significantly increased and Vera $(10 \mu \mathrm{m})$ caused more decrease in co-localization of VPS16/Lamp-1 in Pi-treated CASMCs. $n=3$. (C) Representative 3-D histograms show sEV release from CASMCs. (D) Bar graph shows MLSA-1 decreased sEV release from CASMCs and Vera increased sEV release in these cells. $n=3-4$. Data are shown as mean \pm SEM of values. Phosphate: Pi; Verapamil: Vera. ${ }^{*} p<0.05$ vs. Ctrl Vehl group; $\# p<0.05$ vs. Ctrl Pi group.

\subsection{Mcoln1 Gene Deletion Accelerates Arterial Stiffness}

To address whether the Mcoln 1 gene deletion directly contributes to the development of arterial stiffness, we used Mcoln $1^{-/}$mice. We recently reported that in CASMCs under in vitro conditions, activation of TRPML1 channel enhanced SMC-derived small extracellular vesicles, triggering AMC (unpublished data). PWV is directly correlated with aortic stiffness [36]. Figure 10A shows representative echo images of vehicle and Vit D-treated groups in wild-type and Mcoln $1^{-/-}$mice. As shown in Figure 10B, Mcoln 1 gene deletion increased PWV in Mcoln $1^{-/-}$mice compared with wild-type littermates. To note, Mcoln $1^{-1-}$ mice had increased baseline PWV compared to what we usually observe for healthy control mice, suggesting aortic wall stiffening and remodeling in $M c o l n 1^{-/-}$ even before the development of AMC. Upon receiving high doses of Vit D, we observed a substantial increase in the PWV in the $M c o l n 1^{-/-}$mice as compared to their wild-type littermates, further confirming that the Mcoln1 gene under disturbed blood flow contributes to the arterial stiffness and accelerates the development of AMC. 
One of the characteristics of aortic calcification was disorganization of extracellular matrix and elastin degradation. Elastin staining allowed observation of elastin breaks in aortic sections [37]. Vehicle-treated wild-type aortas displayed normal histological architecture associated with lack of calcification and intact elastic lamellae, which were indistinguishable from vehicle-treated $M c o l n 1^{-/-}$ mice. However, disorganized elastic lamellae with elastin breaks were observed upon Vit D treatment in both wild-type and Mcoln1 $1^{-/-}$mice, as shown in Figure 10C. Due to Mcoln1 gene deletion, elastin breaks and disorganization were significantly increased in $M c o l n 1^{-1-}$ mice as compared to wild-type littermates (Figure 10D), indicating Mcoln1 gene contributes to arterial stiffness associated with elastin remodeling during the development of AMC.

\section{A}
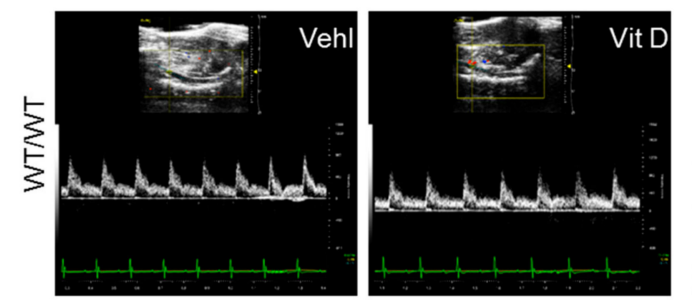

B
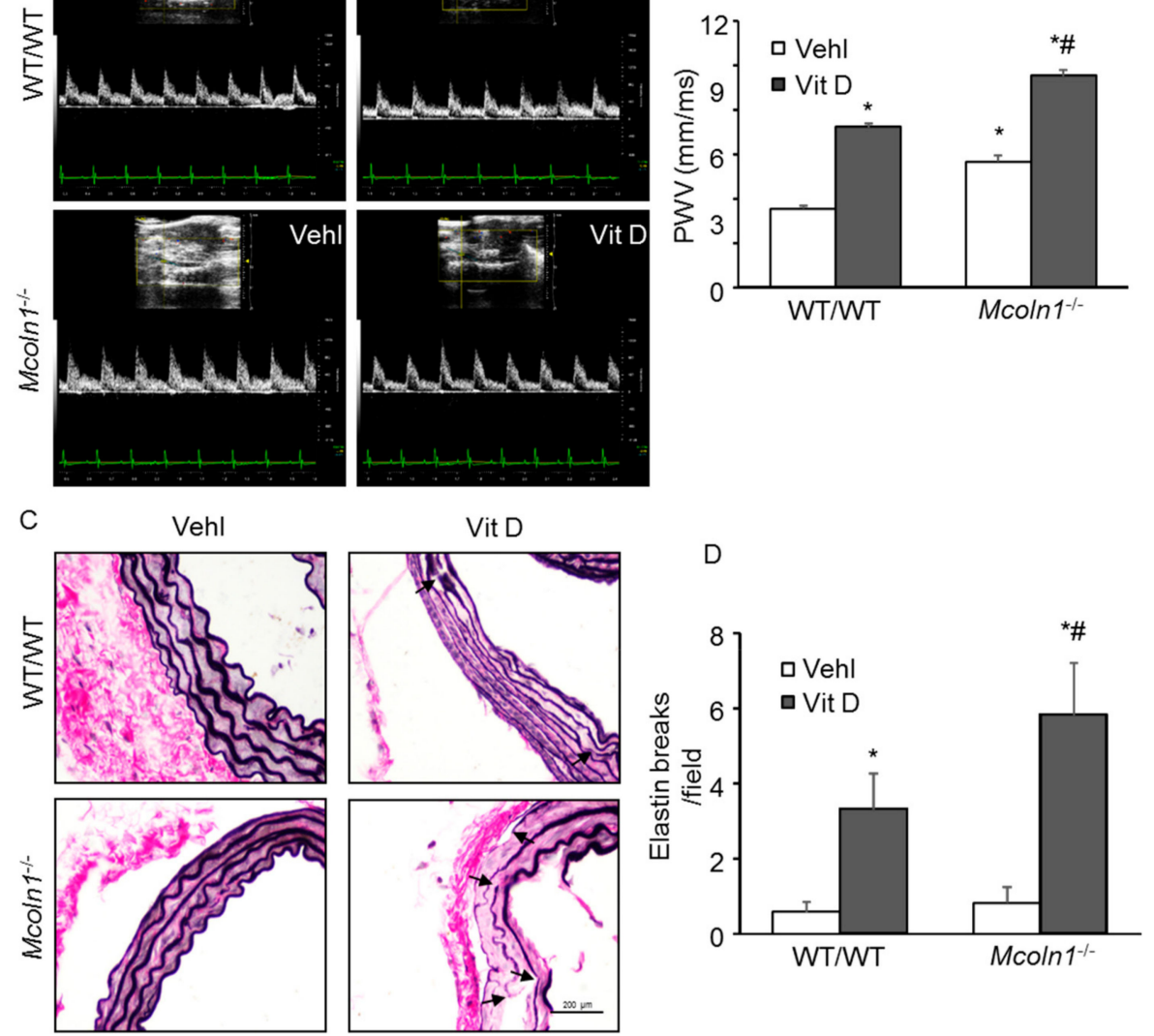

Figure 10. Effect of Mcoln1 gene deletion on arterial stiffness in Vit D-treated Mcoln1-/- mice. (A) Representative Doppler images show Doppler velocity signals. (B) The summarized bar graph shows significantly increased PWV in $M c o l n 1^{-1-}$ mice as compared to wild-type littermates under vehicle conditions as well as with Vit D treatment. $n=5-6$. (C) Representative elastin staining images of aortic sections show breaks in elastic fibers (marked by arrows). (D) The bar diagram shows number of elastin breaks in wild-type and $M \operatorname{coln} 1^{-/-}$mice receiving Vit D injections. Data are shown as mean \pm SEM of values. $n=6$. Vehl: Vehicle; Vit D: vitamin D. ${ }^{*} p<0.05$ vs. WT/WT Vehl; $\# p<0.05$ vs. WT/WT Vit D group.

\section{Discussion}

In the current study, we investigated whether mucolipin-1 (encoded by the Mcoln1 gene) mediates lysosome positioning leading to sEV release in arterial SMCs, contributing to their phenotype switch and arterial stiffness during the development of AMC. First, we found that Mcoln1 gene deletion significantly increased arterial medial calcification in mice treated with a high dose of Vit D. Further, we observed markedly increased immunostaining of osteogenic markers such as "bone" transcription 
factors (e.g., RUNX2) and matrix proteins (e.g., osteopontin) in the arterial medial SMCs of Vit D-treated $M c o l n 1^{-/-}$mice as compared to their wild-type littermates. These results showed that osteogenic shifts in arterial medial SMCs with Mcoln1 gene deletion aid in the development of AMC. We further studied whether Mcoln1 gene deletion alters lysosome positioning, which may result in the release of sEVs. We observed decreased co-localization of lysosome marker (Lamp-1) with lysosome coupling markers (Rab 7 and ALG-2) in the arterial wall of $\mathrm{Mcoln}^{-/-}$mice as compared to their wild-type littermates, suggesting anterograde movement of lysosomes due to Mcoln 1 gene deletion in $M c o l n 1^{-/-}$mice. Also, we observed decreased co-localization of MVBs (VPS16; green) and lysosomes (Lamp-1; red) in the arterial medial SMCs of Mcoln $1^{-1}$ mice as compared to their wild-type littermates associated with increased expression of CD63 and AnX2 (exosome/sEV markers) indicating reduced lysosome-MVB interaction and increased sEV release. In addition, we measured sEVs in the plasma of these mice and found significantly increased plasma sEV in $M c o l n 1^{-/-}$mice as compared to their wild-type littermates. Further, we validated lysosome-MVB interaction and sEV release data in vitro in CASMCs using TRPML1 agonist and blocker in wild-type CASMCs to explore the role of TRPML1 pathway. We found that TRPML1 agonist increased co-localization of VPS16-Lamp-1 and decreased sEV release, whereas TRPML1 blocker showed the opposite trend.

Using a non-invasive ultrasound imaging technique, we observed increased PWV, which is correlated to arterial stiffness due to Mcoln 1 gene deletion in these mice as compared to their littermates. Histopathological architecture showed increased elastin breaks, distorted junctions and formation of solid plates or sheaths in the arterial media of $\mathrm{Mcoln}^{-/-}$mice as compared to their wild- type littermates. Despite our current understanding of the multi-faceted mechanisms regulating AMC, little is known about the lysosomal regulation of sEV release and phenotypic switch of arterial medial SMCs following AMC.

The phenotypic plasticity is an important feature of VSMCs and their contractile phenotype maintains the vascular tone. Upon injury, there is phenotypic transition of these VSMCs from contractile to synthetic. Prolonged exposure of VSMCs to elevated $\mathrm{Ca} / \mathrm{P}_{\mathrm{i}}$ levels induces trans-differentiation of these cells, initiating the formation of osteoblast-like cells, which lead to formation of bone matrix and development of calcification [38-40]. These transdifferentiated VSMCs develop features similar to osteoblasts characterized by the increased expression of bone-related genes osteopontin, osteocalcin and RUNX2, and loss of smooth muscle lineage markers such as SM22- $\alpha[39,41]$. Despite improved understanding of VC pathophysiology, the proper mechanistic pathway leading to production of calcifying extracellular vesicles is still poorly understood. In SMCs, imbalanced mineral metabolism resulted in increased secretion of calcifying sEVs, leading to arterial calcification [8].

TRPML1, also known as Mcoln1, is the primary $\mathrm{Ca}^{2+}$ channel in the lysosome [16,42], which is involved in phagosome lysosome fusion [19], membrane fission from endolysosomes, and autophagosome lysosome fusion [43]. Various TRP channels are known to express in ECs and VSMCs [44,45], which play important roles in the regulation of various vascular disorders. Studies have revealed that TRP channels may contribute to vasoconstriction via receptor activation-induced vascular constriction $[33,46]$. It has been reported that various TRP channels are involved in phenotype switching of SMCs and enhance their proliferation [47-49]. Here, we demonstrated that Mcoln 1 gene deletion increased expression of osteogenic markers such as OSP and RUNX2, whereas the expression of contractile marker SM22- $\alpha$ was markedly decreased in mice injected with high doses of Vit D, indicating the phenotypic transition of arterial medial SMCs.

Lysosome movement towards the cell periphery is required for exosome secretion, which may contribute to the development of VC [27]. Lysosome distribution throughout the cell is either immobile perinuclear near the microtubule-organizing center (MTOC) or highly dynamic peripheral, reaching as far as the plasma membrane [50]. These spatially distinct pools of lysosomes are involved in different cellular processes to maintain cellular homeostasis through their regular mixing by trafficking and fusion processes [51,52]. Rab 7, a small GTPase, and its downstream effectors are mainly involved in coupling of lysosome to dynein-dynactin. Besides Rab7, the lysosomal Ca ${ }^{2+}$-sensor ALG-2 (also 
known as PDCD6) regulates coupling of lysosomes to dynein-dynactin. ALG-2 promotes interaction of dynein-dynactin with lysosomal $\mathrm{Ca}^{2+}$ channel transient receptor potential mucolipin 1 (Mcoln1 or TRPML1) to regulate centripetal transport of lysosomes essential for lysosome exocytosis in response to changes in $\mathrm{Ca}^{2+}$ levels $[15,27]$. TRPML1 induces perinuclear redistribution of lysosomes and links with ALG-2, which then causes TRPML1-dependent retrograde transport of lysosome. Earlier, we revealed that activation of TRPML1 channel by sphingolipid sphingosine contributes to lysosome trafficking [31]. In the present study, we found that the Mcoln1 gene deletion decreased the co-localization lysosome marker (Lamp-1) with lysosome coupling markers (Rab7 and ALG-2) in the aortic medial SMCs during AMC. All these studies support our data, which indicated that Mcoln1 gene plays a crucial role in the lysosome positioning and its deletion resulted in peripheral distribution of lysosomes, which contributes to the extracellular vesicle release, an important feature in AMC.

In human VSMCs, recent studies revealed that exosomes originated from a subset of late endosomal compartment, MVBs [8]. Lysosomal trafficking and its fusion with late endosomes, MVBs, autophagosomes, and the plasma membrane is controlled by TRPML1, a $\mathrm{Ca}^{2+}$-permeable channel [53]. Trafficking of late endosomes, which are generally considered as MVBs, to the plasma membrane results in the release of exosomes [54]. The abovementioned studies provide support to our recent studies and to the present study. Our findings in podocytes demonstrated that inhibition or activation of the TRPML1 channel modulates the interaction of lysosomes and MVBs, thereby regulating the exosome release from these cells [31]. Also, recently, we found that inhibition of TRPML1 channel activation in coronary arterial SMCs due to deficiency of acid ceramidase may lead to the reduction of lysosome-MVB interaction and consequent prolongation of MVB fate, increasing SMC-derived small extracellular vesicle (sEV) release initiating arterial medial calcification (AMC) [55]. Based on these findings, we hypothesized and found that $M c o l n 1^{-/-}$mice exhibited abnormal lysosome positioning and increased sEV levels in the arterial medial SMCs, which may contribute to the arterial stiffness during the development of AMC.

However, the study in OP9 cells reported that TRPML1 regulated exosome release by mediating lysosomal exocytosis during adipogenesis. Although the study concluded that depletion of TRPML1 in pre-adipocytes resulted in a marked reduction of surface LAMP1, which indicated that the study focused towards the exosomes present in lysosomes [30]. Also, as mentioned, trafficking of late endosomes to the plasma membrane allows the release of exosomes [54]. However, several studies in drug-resistant cancer cells revealed that TRPMLs regulate release of exosomes protected from degradation within lysosomes through lysosomal exocytosis [56,57]. The reason for these disparities may be the various downstream targets of lysosomal $\mathrm{Ca}^{2+}$ such as lysosomal exocytosis by synaptotagmin-VII [58], TFEB activation by calcineurin [59] and ALG-2 required for lysosome motility. Interaction of ALG-2 with the dynactin complex promotes retrograde transport. The particular lysosomal subcellular localization and availability of cofactor or substrate may determine which $\mathrm{Ca}^{2+}$ targets are activated for particular $\mathrm{Ca}^{2+}$ release events [15]. For instance, lysosomal $\mathrm{Ca}^{2+}$ release that is docked to the plasma membrane may activate exocytosis and not the retrograde transport [15]. Therefore, different cells using various mechanistic pathways under diverse pathological conditions may lead to exosome release. Our group revealed that pharmacological inhibition of TRPML1 channel by verapamil significantly increased the exosome release from the podocytes, however its activation in response to ML-SA-1, a potent TRPML channel agonist, markedly decreased exosome release. The study concluded that during podocyte injury, inhibition of TRPML1 channel activity by acid ceramidase deficiency increased podocyte-derived exosome release, which may lead to glomerular damage [31]. Concluding, our data also provide further evidence suggesting that Mcoln1 gene deletion modulated lysosome-MVB fusion. We observed decreased co-localization of lysosome marker (Lamp-1) with MVB marker (VPS16) due to Mcoln1 gene deletion in arterial medial SMCs in the development of AMC.

Next, we investigated whether exosome/sEV secretion triggers VC. Several studies revealed that both intimal and medial VC follow a common mechanistic pathway, namely increased extracellular vesicle secretion, especially exosomes $(40-140 \mathrm{~nm}$ in size) in interstitial space [11,60]. Using fetuin-A 
labelling, Kapustin et al. revealed the origin of calcifying exosomes in VSMC [8]. SMC-derived vesicles have increased expression of CD63 and AnX2, identified as exosome markers [10]. However, understanding of the mechanistic pathway mediating exosome biogenesis and secretion in arterial SMCs is still in infancy. In this study, we also observed CD63 and AnX2 (exosome/sEV markers) were significantly increased in the coronary arterial wall of $M c o l n 1^{-1-}$ mice. Correspondingly, in the plasma of Mcoln 1 gene-deficient mice, we observed increased sEV release, which was markedly enhanced with the Vit D treatment in these mice. These findings suggest that lack of Mcoln1 gene inhibits perinuclear lysosome trafficking, which is necessary for lysosome-MVB interaction. Impaired lysosome-MVB interaction leads to increased SEV release in arterial medial SMCs. This enhanced sEV release due to Mcoln1 deficiency may contribute to AMC via cell-to-cell communication.

Several studies showed that augmented plasma endothelial microparticles (EMPs) are associated with the pathogenesis of cardiovascular diseases [61,62]. Plasma EMPs are identified as independent predictors of long-term cardiovascular events in patients with a high risk for coronary heart disease or diabetes $[63,64]$. The plasma levels of endothelial microparticles (EMPs) were found to be significantly increased in patients with type 2 diabetes associated with hypertension and arterial stiffness. In addition, they found that various other microparticles (MPs) such as platelet-derived MPs, annexin V + MPs, and leukocyte-derived MPs were significantly higher in patients with diabetes as compared to healthy patients $(p<0.05)$. The study concluded that in diabetics not only increased blood pressure was associated with impaired arterial stiffness but elevated plasma EMPs act as potent contributors in the progression of arterial stiffness in type 2 diabetes [65]. During aging, concentric elastin lamellae develop medial calcification observed as hydroxyapatite deposits, which increase arterial stiffness without the presence of inflammatory cells [66,67]. Elastin degradation is one of the prominent features of aortic calcification and VSMC phenotype is maintained by an intact elastin matrix in vivo [5]. It has been suggested that transient receptor potential (TRP) channels through their $\mathrm{Ca}^{2+}$ entry, release, and extrusion mechanisms in membranes regulate VSMC contraction known as vasoconstriction response. Theoretically, $\mathrm{Ca}^{2+}$ influx through these channels may directly activate contractile process, but this needs more experimental evidence [68,69]. Moreover, considering the role of transient receptor potential (TRP) channels in VSMC phenotype switching and vasoconstriction, we tried to find out whether Mcoln1 gene deletion contributes to arterial stiffness in mice [47-49]. In this context, we found that Mcoln1 gene deletion markedly increased Pulse Wave Velocity (PWV) with elastin breaks and disorganization, suggesting that Mcoln1 deficiency that is associated with vasoconstriction and VSMC phenotypic transition may contribute to the arterial stiffness during the development of AMC.

In summary, the present study revealed that $M c o l n 1$ gene deletion in mice may play a significant role in both phenotypic maintenance and increased sEV release. Mcoln1 gene deletion caused abnormal lysosome positioning and increased SEV secretion, which may contribute to the arterial stiffness characteristic of AMC. These findings advance our understanding of the complex mechanistic pathways involved in the development and progression of AMC and can be exploited as a novel therapeutic target for ameliorating this detrimental disease.

\section{Material and Methods}

\subsection{Reagents and Antibodies}

Cholecalciferol (vitamin D3) (C9756, Sigma Aldrich, St. Louis, MO, USA). Mucolipin1 (SC-26269, Dallas, TX, USA), OSP (ab63856, Abcam, Cambridge, MA, USA), RUNX2 (ab23981, Abcam, Cambridge, MA, USA), SM22- $\alpha$ (ab14106, Abcam, Cambridge, MA, USA), VPS16 (Cat. No.17776-1-AP, Protein biotech group, Rosemont, IL, USA), Rab7 (ab137029, Abcam, Cambridge, MA, USA), ALG-2 (PDCD6, A6685, Cummings Park Dr, Woburn, MA, USA.), CD63 (ab216130, Abcam, Cambridge, MA, USA) and annexin-II (AnX2, ab41803, Abcam, Cambridge, MA, USA). Secondary antibodies are Alexa-488 or Alexa-555-labeled (Life technologies, Grand Island, NY, USA). Rat monoclonal anti-mouse Lamp-1 
(ab25245, Abcam, Cambridge, MA, USA). Alizarin Red S Solution (TMS-008-C, EMD Millipore. Burlington, MA, USA) was used for detection of arterial medial calcification.

\subsection{Primary Culture of Mouse CASMCs}

Isolation of mouse CASMCs was described previously [34]. Briefly, CASMCs were cultured in Dulbecco's modified Eagle's medium (DMEM, Gibco Gaithersburg, MD, USA), supplemented with $10 \%$ fetal bovine serum (Gibco) and 1\% penicillin-streptomycin (Gibco Gaithersburg, MD, USA) in humidified $100 \%$ air and $5 \% \mathrm{CO} 2$ mixture at $37{ }^{\circ} \mathrm{C}$. Confluent cells $(70 \%-80 \%)$ were treated with or without high phosphate (Pi) (3 mmol/L) [35] and then incubated with TRMPL1 channel agonist, MLSA-1 (10 $\mu \mathrm{M}$, Sigma-Aldrich Chemicals, St. Louis, MO, USA) and TRMPL1 channel blocker, verapamil (10 $\mu \mathrm{M}$, Cayman Chemical, Ann Arbor, MI, USA) incubated for $24 \mathrm{~h}[31,55]$.

\subsection{Animal Model}

Male C57 BL/6 J (12-14 weeks old) wild-type and Mcoln1 $1^{-/}$mice were employed in the present study. Mice homozygous for the $M \operatorname{coln} 1$ gene $\left(\mathrm{Mcoln}^{-/-}\right)$were generated by crossing mice heterozygous for the Mcoln 1 gene $\left(M c o l n 1^{-/+}\right)$(the heterozygous mice were originally purchased from The Jackson Laboratory, B6.Cg-Mcoln $1 \mathrm{tm} 1$ Sasl/J, Stock No: 027110). The average body weight for the male mice aged 12-14 weeks ranged from $\sim 25-30 \mathrm{~g}$. All mice were grossly normal; the homozygous $\left(\mathrm{Mcoln}^{-1-}\right)$ mice, however, were prone to developing hind-limb paralysis by the age of 28 weeks, and the average life expectancy was 8 months. Heterozygosity and homozygosity within the F1 generation was confirmed using PCR analysis. As seen in Figure $1 \mathrm{~A}, M \operatorname{Mcoln} 1^{-/-}$mice had one positive PCR product at $750 \mathrm{bp}$ for the mutant Mcoln1 gene. Mcoln ${ }^{-/+}$mice had two PCR products: one for the mutant Mcoln1 gene (750 bp) and one for the WT gene (453 bp). WT/WT mice had only the WT gene (453 bp). All the animal procedures were approved by the Institutional Animal Care and Use Committee of Virginia Commonwealth University (19 ${ }^{\text {th }}$ Feb 2018) and were registered under protocol number (IACUC, Protocol No. AM10173). Wild-type and Mcoln1 $1^{-/-}$mice $(n=5-6)$ were further divided into two groups each, and injected with active vitamin D (Vit D) $(500,000 \mathrm{IU} / \mathrm{Kg} / \mathrm{BW} /$ day) or matched vehicle $(5 \%$ $v / v$ ethanol) subcutaneously for 3-4 days. Subsequently, animals were sedated with $2 \%$ isoflurane through a nose cone after completing a treatment period followed by blood collection and plasma isolation, which was further stored at $-80^{\circ} \mathrm{C}$. Animals were sacrificed, heart and aorta were removed, and a part was fixed in 10\% buffered formalin for histopathological and immunostaining analysis. Other parts of the heart and aorta were used for frozen tissue slides and stored at $-80^{\circ} \mathrm{C}$ for dual fluorescence staining and confocal analysis. Vit D-induced mouse model is commonly used for the AMC examination. A high dose of Vit D (500,000 IU/Kg/BW/day) [70] was injected to Normal C57 BL/6 $\mathrm{N}$ and $M \mathrm{Mcoln}^{-/-}$mice for 3-4 days ( $n=5-6$ per group). Vit $\mathrm{D}$ solution was prepared by dissolving vitamin D3 $(66 \mathrm{mg})$ in $200 \mu \mathrm{L}$ of absolute ethanol and mixing with $1.4 \mathrm{~mL}$ of cremophor (Sigma Aldrich, Raleigh, NC, USA), and incubated for $15 \mathrm{~min}$ at room temperature, then dextrose solution (750 $\mathrm{mg}$ of dextrose dissolved in $18.4 \mathrm{~mL}$ of sterilized water) was added and left at room temperature for $15 \mathrm{~min}$. The Vit D solution was stored at $4{ }^{\circ} \mathrm{C}$ for further use and made fresh on alternate days.

\subsection{Alizarin Red S Staining}

Alizarin Red S staining (ARS) has been widely used to detect AMC, as described previously [60]. To process ARS, the artery sections were deparaffinized with alcohol and xylene, followed by three washes of distilled water. Then, the artery sections were stained with 1\% Alizarin Red S solution for $5 \mathrm{~min}$ and microscopically observed for orange-red color. Further, the sections were dehydrated with acetone for $20 \mathrm{~s}$, followed by acetone-xylene (1:1) solution for $20 \mathrm{~s}$ and finally in xylene for $5 \mathrm{~min}$. The sections were then mounted with mounting medium and analyzed under light microscopy. The positive ARS signals showed a reddish color. 


\subsection{Calcium Assay}

Blood concentration of calcium was measured using commercially available kits (ab102505, Abcam, Cambridge, MA, USA) as described by the manufacturer's protocol.

\subsection{Immunohistochemical Analyses}

Immunohistochemistry was performed by following the manufacturer's instructions for vectastain universal kit, peroxidase, RTU (PK-7800, Vector Laboratories, Burlingame, CA, USA). Briefly, paraffin-embedded sections $(5 \mu \mathrm{m})$ of the aorta were deparaffinized and prepared for antigen retrieval followed by quenching of endogenous peroxidase activity by using $3 \% \mathrm{H}_{2} \mathrm{O}_{2}$. For blocking, sections were incubated with $2.5 \%$ horse serum for $1 \mathrm{~h}$ at room temperature, followed by incubation with primary antibodies overnight at $4{ }^{\circ} \mathrm{C}$ against SM22- $\alpha$ (1:1500), RUNX2 (1:300), OSP (1:100), CD63 (1:50) and AnX2 (1:50). Subsequently, sections were incubated with biotinylated secondary antibodies and a streptavidin peroxidase complex. Then sections were developed with chromogen 3,3' Diaminobenzidine (DAB) at RT and rinsed in tap water for $5 \mathrm{~min}$. This rinsing was then followed by counterstaining with hematoxylin for $5 \mathrm{~min}$ and dehydration through several washes of alcohol and xylene. Finally, the slides were cleared and mounted with DPX. Image-Pro Plus 6.0 software (Rockvile, MD, USA) was used to calculate the area percentage of the positive staining [71].

\subsection{Immunofluorescent Staining}

To determine the co-localization of various markers in the arterial medial wall, indirect immunofluorescence staining was performed. In this double-layer method, the unconjugated primary antibody binds to the target and fluorochrome-conjugated secondary antibody directed against the primary antibody. Briefly, frozen aortic sections were fixed in acetone, and then sections were incubated in a blocking buffer for $1 \mathrm{~h}$. Subsequently, aortic sections were incubated with diluted primary antibodies of VPS16 (1:300), Rab7 (1:50), ALG-2 (1:100), and (Lamp-1) (1:200) overnight at $4{ }^{\circ} \mathrm{C}$, followed by fluorochrome-conjugated (either Alexa-488 or Alexa-555-labeled) secondary antibody incubation for $1 \mathrm{~h}$ at room temperature. Sections were then rinsed in washing buffer. Finally, sections were mounted with DAPI mounting medium, and staining was examined under a confocal laser scanning microscope (Fluoview FV1000, Olympus, Tokyo, Japan). The rate of co-localization of Lamp-1 with RAB7, ALG-2, VPS16 in the aortic medial SMCs was expressed by Pearson's Correlation Coefficient (PCC) using Image-Pro Plus 6.0 software (Media Cybernetics, Bethesda, MD, USA). PCC is determined by correlation coefficient, $r(r=-1$ to 0$)$, as mentioned previously [71,72].

\subsection{Nanoparticle Tracking Analysis (NTA)}

Nanoparticle Tracking Analysis (NTA) was used to analyze the sEV size and concentration in the plasma of mice using the light scattering mode of the NanoSight LM10 (Nano Sight Ltd., Enigma Business Park, Amesbury, United Kingdom). Plasma was prepared by high-speed centrifugation from anticoagulated blood of mice followed by isolation of sEVs from plasma. Briefly, plasma/cell culture supernatant was centrifuged at $300 \mathrm{~g}$ at $4{ }^{\circ} \mathrm{C}$ for $10 \mathrm{~min}$ to remove detached cells or debris and then filtered through $0.22 \mu \mathrm{m}$ filters to remove apoptotic bodies and microvesicles. Then subjected to ultracentrifugation at 100,000 $\mathrm{g}$ for $90 \mathrm{~min}$ at $4{ }^{\circ} \mathrm{C}$ (Beckman $70.1 \mathrm{~T} 1 \mathrm{ultracentrifuge} \mathrm{rotator}$ ) [73]. To perform NTA, the sEV pellet was diluted in PBS. Each diluted sample was captured in 5 frames (30 s each) with background level 10, camera level 12, and shutter speed 30. The captured video was analyzed with NTA software (Version 3.2 Build 16), and an average particle size distribution graph was plotted with PRISM software (GraphPad, San Diego, CA, USA) [8].

\subsection{Real Time-PCR Studies}

The mRNA levels of TRPML1 (also known as mucolipin 1 gene), OSP and RUNX2 were determined from aorta in the wild-type and Mcoln $1^{-1-}$ mice by RT-PCR. The following 
mouse-specific primers were used: $\beta$-actin, sense $5^{\prime}$-TCGCTGCGCTGGTCGTC- $3^{\prime}$ and antisense 5'-GGCCTCGTCACCCACATAGGA-3' ; mucolipin-1, sense 5'-ATTTGCTTTTGCCTGTTTGG-3' and antisense $5^{\prime}$-CTCCATCGTCATCATCATCG-3'; OSP, sense 5'-ATTTGCTTTTGCCTGTTTGG-3' and antisense $5^{\prime}$-CTCCATCGTCATCATCATCG-3'; RUNX2 sense 5' -CCAGATGGGACTGTGGTTACC-3' and antisense $5^{\prime}$-ACTTGGTGCAGAGTTCAGGG-3'. $\beta$-Actin was used as anternal control to normalize the expression of mRNA. Fold changes were calculated as follows: $2^{-\Delta \Delta}$ threshold cycle [74].

\subsection{Western Blot Analysis}

Briefly, SDS-PAGE was used to resolve equal amounts of protein isolated from the aorta along with the molecular weight marker, transferred to PVDF membrane. Milk (5\%) was used for blocking the membrane, followed by incubation with respective primary antibodies rabbit polyclonal to SM22- $\alpha$ (1:10,000, abcam, Cambridge, MA, USA), rabbit polyclonal to RUNX2 (1:1000, abcam, USA) and rabbit anti- $\beta$-actin (1:10,000, Santa Cruz Biotechnology, Dallas, TX, USA) overnight at $4{ }^{\circ} \mathrm{C}$. Next day incubated with donkey anti-rabbit-HRP IgG (1:5000, Santa Cruz Biotechnology, Dallas, TX, USA) for $1 \mathrm{~h}$ at room temperature. Finally, bands were detected by chemiluminescence technique using LI-COR Odyssey Fc and the band intensity of target proteins was normalized to $\beta$-actin and calculated with Image J software version $1.44 \mathrm{p}$ (NIH, Bethesda, MD, USA) [71].

\subsection{Non-Invasive, in Vivo Measurement of Aortic Pulse Wave Velocity (PWV)}

Pulse Wave Velocity (PWV) was used to measure arterial stiffness post-Vit D treatment by using a high-resolution Doppler ultrasound instrument (Vevo2100, Visual-Sonics, Toronto, ON, Canada) [75]. Briefly, animals were anesthetized with $2 \%$ isoflurane and then mounted on a temperature-controlled platform where the body temperature of mice was maintained at $37^{\circ} \mathrm{C}$ to remove the movement artifacts. Different parameters, such as an electrocardiogram (ECG), heart rate (HR), and respiratory rate were then observed. In addition, heart rate (HR) was maintained around $450 \mathrm{bpm}$ by adjusting isoflurane concentration to $1.5 \%$. Flow wave Doppler measurement of aortic outflow was captured with a $6 \mathrm{~mm}, 30 \mathrm{MHz}$ pulsed Doppler probe with a pulse repetition frequency of $40 \mathrm{kHz}$. Aortic outflow profiles were captured longitudinally at two locations, one proximal and the other distal to the heart. Heart rate was similar in both proximal and distal point measurements. PWV was estimated at both the proximal and distal locations from the peak of the ECG R wave to the foot of the flow waves for 5 replicates in each mouse. PWV $(\mathrm{mm} / \mathrm{ms})$ was calculated using the TT $(\Delta \mathrm{t})$ and the distance between the measurement sites $(\Delta \mathrm{d})$, where TT (Transit time) is the difference between the time of arrival at proximal and distal points [75].

\subsection{Elastin Staining}

Elastin staining or Verhoeff's Van Gieson stain was used to stain elastic fibers in aortic tissue sections. Staining was performed by following manufacturer's instructions (ab150667, Abcam, Cambridge, MA, USA). Briefly, sections were deparaffinized, then hydrated in distilled water. Sections were then incubated in elastin stain solution for $15 \mathrm{~min}$, rinsed in running tap water to remove excess stain. Further, sections were dipped in differentiating solution 15-20 times, rinsed in tap water, and checked under the microscope for proper differentiation. Then, sections were incubated in sodium thiosulfate solution for $1 \mathrm{~min}$, rinsed in running water, followed by staining of sections using Van Gieson solution for 2-5 min. Lastly, sections were washed in 95\% alcohol 2 times, dehydrated in absolute alcohol, and mounted in synthetic resin.

\subsection{Statistical Analysis}

All of the values are expressed as mean \pm SEM. One-way and two-way ANOVA was used to examine the significant differences among multiple groups, followed by a Tukey's or Duncan's test. $p<0.05$ was considered statistically significant. 
Author Contributions: O.M.B. and P.-L.L. planned and designed the studies. F.N.S. provided the technical facility for various experiments. S.C. characterized and maintained KO mice. O.M.B and X.Y. conducted experiments and generated data. O.M.B. analyzed and interpreted data. O.M.B. and P.-L.L. wrote and revised the manuscript. All authors approved the final version of the manuscript.

Acknowledgments: This study was supported by grants from the National Institutes of Health (HL057244, HL075316 and DK120491).

Conflicts of Interest: The authors declare no competing financial and non-financial interests.

\section{References}

1. Speer, M.Y.; Giachelli, C.M. Regulation of cardiovascular calcification. Cardiovasc. Pathol. 2004, 13, 63-70. [CrossRef]

2. Giachelli, C.M. Mechanisms of vascular calcification in uremia. Semin. Nephrol. 2004, 24, 401-402. [CrossRef] [PubMed]

3. Burke, A.P.; Taylor, A.; Farb, A.; Malcom, G.T.; Virmani, R. Coronary calcification: insights from sudden coronary death victims. Z. Kardiol. 2000, 89, 49-53. [CrossRef] [PubMed]

4. Edmonds, M.E.; Morrison, N.; Laws, J.W.; Watkins, P.J. Medial arterial calcification and diabetic neuropathy. Br. Med. J. (Clin Res. Ed.). 1982, 284, 928-930. [CrossRef] [PubMed]

5. Demer, L.L.; Tintut, Y. Vascular calcification: Pathobiology of a multifaceted disease. Circulation 2008, 117, 2938-2948. [CrossRef] [PubMed]

6. $\quad$ Reynolds, J.L.; Joannides, A.J.; Skepper, J.N.; McNair, R.; Schurgers, L.J.; Proudfoot, D.; Jahnen Dechent, W.; Weissberg, P.L.; Shanahan, C.M. Human vascular smooth muscle cells undergo vesicle-mediated calcification in response to changes in extracellular calcium and phosphate concentrations: A potential mechanism for accelerated vascular calcification in ESRD. J. Am. Soc. Nephrol. 2004, 15, 2857-2867. [PubMed]

7. Tkach, M.; Thery, C. Communication by Extracellular Vesicles: Where We Are and Where We Need to Go. Cell 2016, 164, 1226-1232. [CrossRef]

8. Kapustin, A.N.; Chatrou, M.L.; Drozdov, I.; Zheng, Y.; Davidson, S.M.; Soong, D.; Furmanik, M.; Sanchis, P.; De Rosales, R.T.M.; Alvarez-Hernandez, D.; et al. Vascular smooth muscle cell calcification is mediated by regulated exosome secretion. Circ. Res. 2015, 116, 1312-1323. [CrossRef]

9. Kapustin, A.N.; Schoppet, M.; Schurgers, L.J.; Reynolds, J.L.; McNair, R.; Heiss, A.; Jahnen-Dechent, W.; Hackeng, T.M.; Schlieper, G.; Harrison, P.; et al. Prothrombin Loading of Vascular Smooth Muscle Cell-Derived Exosomes Regulates Coagulation and Calcification. Arterioscler. Thromb. Vasc. Biol. 2017, 37, e22-e32. [CrossRef]

10. Chen, N.X.; O'Neill, K.D.; Moe, S.M. Matrix vesicles induce calcification of recipient vascular smooth muscle cells through multiple signaling pathways. Kidney Int. 2018, 93, 343-354. [CrossRef]

11. Kapustin, A.N.; Shanahan, C.M. Emerging roles for vascular smooth muscle cell exosomes in calcification and coagulation. J. Physiol. 2016, 594, 2905-2914. [CrossRef] [PubMed]

12. Zeidan, Y.H.; Jenkins, R.W.; Hannun, Y.A. Remodeling of cellular cytoskeleton by the acid sphingomyelinase/ ceramide pathway. J. Cell Biol. 2008, 181, 335-350. [CrossRef] [PubMed]

13. Saftig, P.; Klumperman, J. Lysosome biogenesis and lysosomal membrane proteins: trafficking meets function. Nat. Rev. Mol. Cell Biol. 2009, 10, 623-635. [CrossRef]

14. Luzio, J.P.; Pryor, P.R.; Bright, N.A. Lysosomes: fusion and function. Nat. Rev. Mol. Cell Biol. 2007, 8, 622-632. [CrossRef] [PubMed]

15. Li, X.; Rydzewski, N.; Hider, A.; Zhang, X.; Yang, J.; Wang, W.; Gao, Q.; Cheng, X.; Xu, H. A molecular mechanism to regulate lysosome motility for lysosome positioning and tubulation. Nat. Cell Biol. 2016, 18, 404-417. [CrossRef] [PubMed]

16. Cheng, X.; Shen, D.; Samie, M.; Xu, H. Mucolipins: Intracellular TRPML1-3 channels. FEBS Lett. 2010, 584, 2013-2021. [CrossRef]

17. Shen, D.; Wang, X.; Xu, H. Pairing phosphoinositides with calcium ions in endolysosomal dynamics: phosphoinositides control the direction and specificity of membrane trafficking by regulating the activity of calcium channels in the endolysosomes. Bioessays 2011, 33, 448-457. [CrossRef] 
18. Xu, M.; Li, X.; Walsh, S.W.; Zhang, Y.; Abais, J.M.; Boini, K.M.; Li, P.L. Intracellular two-phase Ca2+ release and apoptosis controlled by TRP-ML1 channel activity in coronary arterial myocytes. Am. J. Physiol. Cell Physiol. 2013, 304, C458-C466. [CrossRef]

19. Dayam, R.M.; Saric, A.; Shilliday, R.E.; Botelho, R.J. The Phosphoinositide-Gated Lysosomal Ca (2+) Channel, TRPML1, Is Required for Phagosome Maturation. Traffic 2015, 16, 1010-1026. [CrossRef]

20. Lee, J.H.; McBrayer, M.K.; Wolfe, D.M.; Haslett, L.J.; Kumar, A.; Sato, Y.; Lie, P.P.Y.; Mohan, P.; Coffey, E.E.; Kompella, U.; et al. Presenilin 1 Maintains Lysosomal Ca(2+) Homeostasis via TRPML1 by Regulating vATPase-Mediated Lysosome Acidification. Cell Rep. 2015, 12, 1430-1444. [CrossRef]

21. Chen, C.S.; Bach, G.; Pagano, R.E. Abnormal transport along the lysosomal pathway in mucolipidosis, type IV disease. Proc. Natl. Acad. Sci. USA 1998, 95, 6373-6378. [CrossRef] [PubMed]

22. Miller, A.; Schafer, J.; Upchurch, C.; Spooner, E.; Huynh, J.; Hernandez, S.; McLaughlin, B.; Oden, L.; Fares, H. Mucolipidosis type IV protein TRPML1-dependent lysosome formation. Traffic 2015, 16, 284-297. [CrossRef] [PubMed]

23. Treusch, S.; Knuth, S.; Slaugenhaupt, S.A.; Goldin, E.; Grant, B.D.; Fares, H. Caenorhabditis elegans functional orthologue of human protein h-mucolipin-1 is required for lysosome biogenesis. Proc. Natl. Acad. Sci. USA 2004, 101, 4483-4488. [CrossRef]

24. Bargal, R.; Bach, G. Mucolipidosis type IV: abnormal transport of lipids to lysosomes. J. Inherit. Metab. Dis. 1997, 20, 625-632. [CrossRef]

25. Thompson, E.G.; Schaheen, L.; Dang, H.; Fares, H. Lysosomal trafficking functions of mucolipin-1 in murine macrophages. BMC Cell Biol. 2007, 8, 54. [CrossRef]

26. Kiselyov, K.; Chen, J.; Rbaibi, Y.; Oberdick, D.; Tjon-Kon-Sang, S.; Shcheynikov, N.; Muallem, S.; Soyombo, A. TRP-ML1 is a lysosomal monovalent cation channel that undergoes proteolytic cleavage. J. Biol. Chem. 2005, 280, 43218-43223. [CrossRef]

27. Pu, J.; Guardia, C.M.; Keren-Kaplan, T.; Bonifacino, J.S. Mechanisms and functions of lysosome positioning. J. Cell Sci. 2016, 129, 4329-4339. [CrossRef]

28. Futter, C.E.; Pearse, A.; Hewlett, L.J.; Hopkins, C.R. Multivesicular endosomes containing internalized EGF-EGF receptor complexes mature and then fuse directly with lysosomes. J. Cell Biol. 1996, 132, 1011-1023. [CrossRef]

29. Hessvik, N.P.; Llorente, A. Current knowledge on exosome biogenesis and release. Cell Mol. Life Sci. 2018, 75, 193-208. [CrossRef]

30. Kim, M.S.; Muallem, S.; Kim, S.H.; Kwon, K.B.; Kim, M.S. Exosomal release through TRPML1-mediated lysosomal exocytosis is required for adipogenesis. Biochem. Biophys Res. Commun. 2019, 510, 409-415. [CrossRef]

31. Li, G.; Huang, D.; Hong, J.; Bhat, O.M.; Yuan, X.; Li, P.L. Control of lysosomal TRPML1 channel activity and exosome release by acid ceramidase in mouse podocytes. Am. J. Physiol. Cell Physiol. 2019, 317, C481-C491. [CrossRef] [PubMed]

32. Hutcheson, J.D.; Goettsch, C.; Bertazzo, S.; Maldonado, N.; Ruiz, J.L.; Goh, W.; Yabusaki, K.; Faits, T.; Bouten, C.; Franck, G.; et al. Genesis and growth of extracellular-vesicle-derived microcalcification in atherosclerotic plaques. Nat. Mater. 2016, 15, 335-343. [CrossRef] [PubMed]

33. Yue, Z.; Xie, J.; Yu, A.S.; Stock, J.; Du, J.; Yue, L. Role of TRP channels in the cardiovascular system. Am. J. Physiol. Heart Circ. Physiol. 2015, 308, H157-H182. [CrossRef] [PubMed]

34. Shanahan, C.M.; Crouthamel, M.H.; Kapustin, A.; Giachelli, C.M. Arterial calcification in chronic kidney disease: key roles for calcium and phosphate. Circ. Res. 2011, 109, 697-711. [CrossRef] [PubMed]

35. Akers, J.C.; Gonda, D.; Kim, R.; Carter, B.S.; Chen, C.C. Biogenesis of extracellular vesicles (EV): exosomes, microvesicles, retrovirus-like vesicles, and apoptotic bodies. J. Neurooncol. 2013, 113, 1-11. [CrossRef] [PubMed]

36. Lane, H.A.; Smith, J.C.; Davies, J.S. Noninvasive assessment of preclinical atherosclerosis. Vasc. Health Risk Manag. 2006, 2, 19-30. [CrossRef]

37. Ju, X.; Ijaz, T.; Sun, H.; Lejeune, W.; Vargas, G.; Shilagard, T.; RecinosIII, A.; Milewicz, D.M.; Brasier, A.R.; Tilton, R.G. IL-6 regulates extracellular matrix remodeling associated with aortic dilation in a fibrillin-1 hypomorphic mgR/mgR mouse model of severe Marfan syndrome. J. Am. Heart Assoc. 2014, 3, e000476. [CrossRef] 
38. Katoh, Y.; Periasamy, M. Growth and differentiation of smooth muscle cells during vascular development. Trends Cardiovasc. Med. 1996, 6, 100-106. [CrossRef]

39. Giachelli, C.M.; Liaw, L.; Murry, C.E.; Schwartz, S.M.; Almeida, M. Osteopontin expression in cardiovascular diseases. Ann. N. Y. Acad Sci. 1995, 760, 109-126. [CrossRef]

40. Steitz, S.A.; Speer, M.Y.; Curinga, G.; Yang, H.Y.; Haynes, P.; Aebersold, R.; Schinke, T.; Karsenty, G.; Giachelli, C.M. Smooth muscle cell phenotypic transition associated with calcification: upregulation of Cbfa1 and downregulation of smooth muscle lineage markers. Circ. Res. 2001, 89, 1147-1154. [CrossRef]

41. Shroff, R.C.; McNair, R.; Figg, N.; Skepper, J.N.; Schurgers, L.; Gupta, A.; Hiorns, M.; Donald, A.E.; Deanfield, J.; Rees, J.; et al. Dialysis accelerates medial vascular calcification in part by triggering smooth muscle cell apoptosis. Circulation. 2008, 118, 1748-1757. [CrossRef] [PubMed]

42. Dong, X.P.; Cheng, X.; Mills, E.; Delling, M.; Wang, F.; Kurz, T.; Xu, H. The type IV mucolipidosis-associated protein TRPML1 is an endolysosomal iron release channel. Nature 2008, 455, 992-996. [CrossRef] [PubMed]

43. Xu, H.; Ren, D. Lysosomal physiology. Annu. Rev. Physiol. 2015, 77, 57-80. [CrossRef] [PubMed]

44. Dietrich, A.; Chubanov, V.; Kalwa, H.; Rost, B.R.; Gudermann, T. Cation channels of the transient receptor potential superfamily: their role in physiological and pathophysiological processes of smooth muscle cells. Pharmacol. Ther. 2006, 112, 744-760. [CrossRef]

45. Sullivan, M.N.; Earley, S. TRP channel $\mathrm{Ca}(2+)$ sparklets: fundamental signals underlying endothelium-dependent hyperpolarization. Am. J. Physiol. Cell Physiol. 2013, 305, C999-C1008. [CrossRef]

46. Dietrich, A.; Mederos, Y.S.M.; Gollasch, M.; Gross, V.; Storch, U.; Dubrovska, G.; Obst, M.; Yildirim, E.; Salanova, B.; Kalwa, H.; et al. Increased vascular smooth muscle contractility in TRPC6-/- mice. Mol. Cell Biol. 2005, 25, 6980-6989. [CrossRef]

47. Bergdahl, A.; Gomez, M.F.; Wihlborg, A.K.; Erlinge, D.; Eyjolfson, A.; Xu, S.Z.; Beech, D.J.; Dreja, K.; Hellstrand, P. Plasticity of TRPC expression in arterial smooth muscle: Correlation with store-operated Ca2+ entry. Am. J. Physiol. Cell Physiol. 2005, 288, C872-C880. [CrossRef]

48. Kumar, B.; Dreja, K.; Shah, S.S.; Cheong, A.; Xu, S.Z.; Sukumar, P.; Naylor, J.; Forte, A.; Cipollaro, M.; McHugh, D.; et al. Upregulated TRPC1 channel in vascular injury in vivo and its role in human neointimal hyperplasia. Circ. Res. 2006, 98, 557-563. [CrossRef]

49. Takahashi, Y.; Watanabe, H.; Murakami, M.; Ohba, T.; Radovanovic, M.; Ono, K.; Iijima, T.; Ito, H. Involvement of transient receptor potential canonical 1 (TRPC1) in angiotensin II-induced vascular smooth muscle cell hypertrophy. Atherosclerosis 2007, 195, 287-296. [CrossRef]

50. Jongsma, M.L.; Berlin, I.; Wijdeven, R.H.; Janssen, L.; Janssen, G.M.; Garstka, M.A.; Janssen, H.; Mensink, M.; van Veelen, P.A.; Spaapen, R.M.; et al. An ER-Associated Pathway Defines Endosomal Architecture for Controlled Cargo Transport. Cell. 2016, 166, 152-166. [CrossRef]

51. Cabukusta, B.; Neefjes, J. Mechanisms of lysosomal positioning and movement. Traffic. 2018, 19, 761-769. [CrossRef] [PubMed]

52. Jordens, I.; Fernandez-Borja, M.; Marsman, M.; Dusseljee, S.; Janssen, L.; Calafat, J.; Janssen, H.; Wubbolts, R.; Neefjes, J. The Rab7 effector protein RILP controls lysosomal transport by inducing the recruitment of dynein-dynactin motors. Curr. Biol. 2001, 11, 1680-1685. [CrossRef]

53. Wang, W.; Zhang, X.; Gao, Q.; Xu, H. TRPML1: an ion channel in the lysosome. Handb. Exp. Pharmacol. 2014, 222, 631-645. [PubMed]

54. Kowal, J.; Tkach, M.; Thery, C. Biogenesis and secretion of exosomes. Curr. Opin. Cell Biol. 2014, $29,116-125$. [CrossRef] [PubMed]

55. Bhat, O.M.; Li, G.; Yuan, X.; Huang, D.; Gulbins, E.; Kukreja, R.C.; Li, P.L. Arterial Medial Calcification through Enhanced small Extracellular Vesicle Release in Smooth Muscle-Specific Asah1 Gene Knockout Mice. Sci. Rep. 2020, 10, 1645. [CrossRef] [PubMed]

56. Zhitomirsky, B.; Assaraf, Y.G. Lysosomal accumulation of anticancer drugs triggers lysosomal exocytosis. Oncotarget 2017, 8, 45117-45132. [CrossRef]

57. Miao, Y.; Li, G.; Zhang, X.; Xu, H.; Abraham, S.N. A TRP Channel Senses Lysosome Neutralization by Pathogens to Trigger Their Expulsion. Cell 2015, 161, 1306-1319. [CrossRef]

58. Samie, M.A.; Xu, H. Lysosomal exocytosis and lipid storage disorders. J. Lipid Res. 2014, 55, 995-1009. [CrossRef] 
59. Medina, D.L.; Di Paola, S.; Peluso, I.; Armani, A.; De Stefani, D.; Venditti, R.; Montefusco, S.; Scotto-Rosato, A.; Prezioso, C.; Forrester, A.; et al. Lysosomal calcium signalling regulates autophagy through calcineurin and TFEB. Nat. Cell Biol. 2015, 17, 288-299. [CrossRef]

60. Bhat, O.M.; Yuan, X.; Cain, C.; Salloum, F.N.; Li, P.L. Medial calcification in the arterial wall of smooth muscle cell-specific Smpd1 transgenic mice: A ceramide-mediated vasculopathy. J. Cell Mol. Med. 2020, 24, 539-553. [CrossRef]

61. Agouni, A.; Lagrue-Lak-Hal, A.H.; Ducluzeau, P.H.; Mostefai, H.A.; Draunet-Busson, C.; Leftheriotis, G.; Heymes, C.; Martinez, M.C.; Andriantsitohaina, R. Endothelial dysfunction caused by circulating microparticles from patients with metabolic syndrome. Am. J. Pathol. 2008, 173, 1210-1219. [CrossRef] [PubMed]

62. Wang, J.M.; Su, C.; Wang, Y.; Huang, Y.J.; Yang, Z.; Chen, L.; Wu, F.; Xu, S.Y.; Tao, J. Elevated circulating endothelial microparticles and brachial-ankle pulse wave velocity in well-controlled hypertensive patients. J. Hum. Hypertens. 2009, 23, 307-315. [CrossRef] [PubMed]

63. Nozaki, T.; Sugiyama, S.; Koga, H.; Sugamura, K.; Ohba, K.; Matsuzawa, Y.; Sumida, H.; Matsui, K.; Jinnouchi, H.; Ogawa, H. Significance of a multiple biomarkers strategy including endothelial dysfunction to improve risk stratification for cardiovascular events in patients at high risk for coronary heart disease. J. Am. Coll. Cardiol. 2009, 54, 601-608. [CrossRef]

64. Koga, H.; Sugiyama, S.; Kugiyama, K.; Watanabe, K.; Fukushima, H.; Tanaka, T.; Sakamoto, T.; Yoshimura, W.; Jinnouchi, H.; Ogawa, H. Elevated levels of VE-cadherin-positive endothelial microparticles in patients with type 2 diabetes mellitus and coronary artery disease. J. Am. Coll. Cardiol. 2005, 45, 1622-1630. [CrossRef]

65. Chen, Y.; Feng, B.; Li, X.; Ni, Y.; Luo, Y. Plasma endothelial microparticles and their correlation with the presence of hypertension and arterial stiffness in patients with type 2 diabetes. J. Clin. Hypertens (Greenwich). 2012, 14, 455-460. [CrossRef]

66. Shroff, R.C.; Shanahan, C.M. The vascular biology of calcification. Semin. Dial. 2007, 20, 103-109. [CrossRef]

67. Mackey, R.H.; Venkitachalam, L.; Sutton-Tyrrell, K. Calcifications, arterial stiffness and atherosclerosis. Adv. Cardiol. 2007, 44, 234-244.

68. Earley, S.; Brayden, J.E. Transient receptor potential channels in the vasculature. Physiol. Rev. 2015, 95, 645-690. [CrossRef]

69. Tu, H.; Zhang, D.; Li, Y.L. Cellular and Molecular Mechanisms Underlying Arterial Baroreceptor Remodeling in Cardiovascular Diseases and Diabetes. Neurosci. Bull. 2019, 35, 98-112. [CrossRef]

70. Price, P.A.; Buckley, J.R.; Williamson, M.K. The amino bisphosphonate ibandronate prevents vitamin D toxicity and inhibits vitamin D-induced calcification of arteries, cartilage, lungs and kidneys in rats. J. Nutr. 2001, 131, 2910-2915. [CrossRef]

71. Yuan, X.; Bhat, O.M.; Meng, N.; Lohner, H.; Li, P.L. Protective Role of Autophagy in Nlrp3 Inflammasome Activation and Medial Thickening of Mouse Coronary Arteries. Am. J. Pathol. 2018, 188, 2948-2959. [CrossRef] [PubMed]

72. La Rosa, L.R.; Perrone, L.; Nielsen, M.S.; Calissano, P.; Andersen, O.M.; Matrone, C. Y682 G Mutation of Amyloid Precursor Protein Promotes Endo-Lysosomal Dysfunction by Disrupting APP-SorLA Interaction. Front. Cell Neurosci. 2015, 9, 109. [CrossRef] [PubMed]

73. Yuan, X.; Bhat, O.M.; Lohner, H.; Zhang, Y.; Li, P.L. Endothelial acid ceramidase in exosome-mediated release of NLRP3 inflammasome products during hyperglycemia: Evidence from endothelium-specific deletion of Asah1 gene. Biochim. Biophys Acta Mol. Cell Biol. Lipids. 2019, 1864, 158532. [CrossRef] [PubMed]

74. Yuan, X.; Bhat, O.M.; Lohner, H.; Li, N.; Zhang, Y.; Li, P.L. Inhibitory effects of growth differentiation factor 11 on autophagy deficiency-induced dedifferentiation of arterial smooth muscle cells. Am. J. Physiol. Heart Circ. Physiol. 2019, 316, H345-H356. [CrossRef] [PubMed]

75. Tong, X.; Khandelwal, A.R.; Wu, X.; Xu, Z.; Yu, W.; Chen, C.; Zhao, W.; Yang, J.; Qin, Z.; Weisbrod, R.M.; et al. Pro-atherogenic role of smooth muscle Nox4-based NADPH oxidase. J. Mol. Cell Cardiol. 2016, 92, 30-40. [CrossRef]

(C) 2020 by the authors. Licensee MDPI, Basel, Switzerland. This article is an open access article distributed under the terms and conditions of the Creative Commons Attribution (CC BY) license (http://creativecommons.org/licenses/by/4.0/). 